\title{
An Experimental Analysis of Parallel Sorting Algorithms*
}

\author{
G. E. Blelloch, ${ }^{1}$ C. E. Leiserson, ${ }^{2}$ B. M. Maggs, ${ }^{1}$ C. G. Plaxton, ${ }^{3}$
}

S. J. Smith, ${ }^{4}$ and M. Zagha ${ }^{5}$

${ }^{1}$ Carnegie Mellon University,

Pittsburgh, PA 15213, USA

${ }^{2}$ MIT, Cambridge,

MA 02139, USA

${ }^{3}$ University of Texas,

Austin, TX 78712, USA

${ }^{4}$ Pilot Software,

Cambridge, MA 02141, USA

${ }^{5}$ Silicon Graphics, Mountain View,

CA 94043, USA

\begin{abstract}
We have developed a methodology for predicting the performance of parallel algorithms on real parallel machines. The methodology consists of two steps. First, we characterize a machine by enumerating the primitive operations that it is capable of performing along with the cost of each operation. Next, we analyze an algorithm by making a precise count of the number of times the algorithm performs each type of operation. We have used this methodology to evaluate many of the parallel sorting algorithms proposed in the literature. Of these, we selected the three most promising, Batcher's bitonic sort, a parallel radix sort, and a sample sort similar to Reif and Valiant's flashsort, and implemented them on the connection Machine model CM-2. This paper analyzes the three algorithms in detail and discusses the issues that led us to our particular implementations. On the CM-2 the predicted performance of the algorithms closely matches the observed performance, and hence our methodology can be used to tune the algorithms for optimal performance. Although our programs were designed for the CM-2, our conclusions about the merits of the three algorithms apply to other parallel machines as well.
\end{abstract}

* This research was supported in part by Thinking Machines Corporation, and in part by the Defense Advanced Research Projects Agency under Contracts N00014-87-0825 and F33615-90-C-1465. CM-2, Connection Machine, CM, *Lisp, Paris, and CMIS are trademarks of Thinking Machines Corporation. 


\section{Introduction}

Sorting is arguably the most studied problem in computer science, both because it is used as a substep in many applications and because it is a simple combinatorial problem with many interesting and diverse solutions. Sorting is also an important benchmark for parallel supercomputers. It requires significant communication bandwidth among processors, unlike many other supercomputer benchmarks, and the most efficient sorting algorithms communicate data in irregular patterns.

Parallel algorithms for sorting have been studied since at least the 1960s. An early advance in parallel sorting came in 1968 when Batcher discovered the elegant bitonic sorting network [3] which sorts $n$ keys in depth $(\lg n)(\lg n+1) / 2$. (Throughout this paper $\lg n$ denotes $\log _{2} n$.) For certain families of fixed interconnection networks, such as the hypercube and shuffle-exchange, Batcher's bitonic sorting technique provides a parallel algorithm for sorting $n$ numbers in $\Theta\left(\lg ^{2} n\right)$ time with $n$ processors. The question of the existence of a $o\left(\lg ^{2} n\right)$-depth sorting network remained open until 1983, when Ajtai, Komlós, and Szemerédi [1] provided an optimal $\Theta(\lg n)$-depth sorting network, but, unfortunately, their construction leads to larger networks than those given by bitonic sort for all "practical" values of $n$. Leighton [15] has shown that any $\Theta(\lg n)$-depth family of sorting networks can be used to sort $n$ numbers in $\Theta(\lg n)$ time in the $n$-node boundeddegree fixed-connection network domain. Not surprisingly, the optimal $\Theta(\lg n)$-time $n$-node fixed-connection sorting networks implied by the AKS construction are also impractical.

In 1983 Reif and Valiant proposed a more practical $O(\lg n)$-time randomized algorithm for sorting [19], called flashsort. Many other parallel sorting algorithms have been proposed in the literature, including parallel versions of radix sort and quicksort [5], a variant of quicksort called hyperquicksort [23], smoothsort [18], column sort [15], Nassimi and Sahni's sort [17], and parallel merge sort [6].

This paper reports the findings of a project undertaken at Thinking Machines Corporation to develop a fast sorting algorithm for the Connection Machine Supercomputer model CM-2. The primary goals of this project were:

1. To implement as fast a sorting algorithm as possible for integers and floating-pont numbers on the CM-2.

2. To generate a library sort for the CM-2 (here we were concerned with memory use, stability, and performance over a wide range of problem and key sizes in addition to running time).

3. To gain insight into practical sorting algorithms in general.

Our first step toward achieving these goals was to analyze and evaluate many of the parallel sorting algorithms that have been proposed in the literature. After analyzing many algorithms, we selected the three most promising alternatives for implementation: bitonic sort, radix sort, and sample sort. Figure 1 compares the running times of these three algorithms (two versions of radix sort are shown). Typically the number of keys, $n$, is larger than the number of processors, $p$. As is apparent from the figure, when the number of keys per processor $(n / p)$ is large, sample sort is the fastest sorting algorithm. On the other hand, radix sort performs reasonably well over the entire range of $n / p$, and it is deterministic, much simpler to code, stable, and faster with small keys. Although 


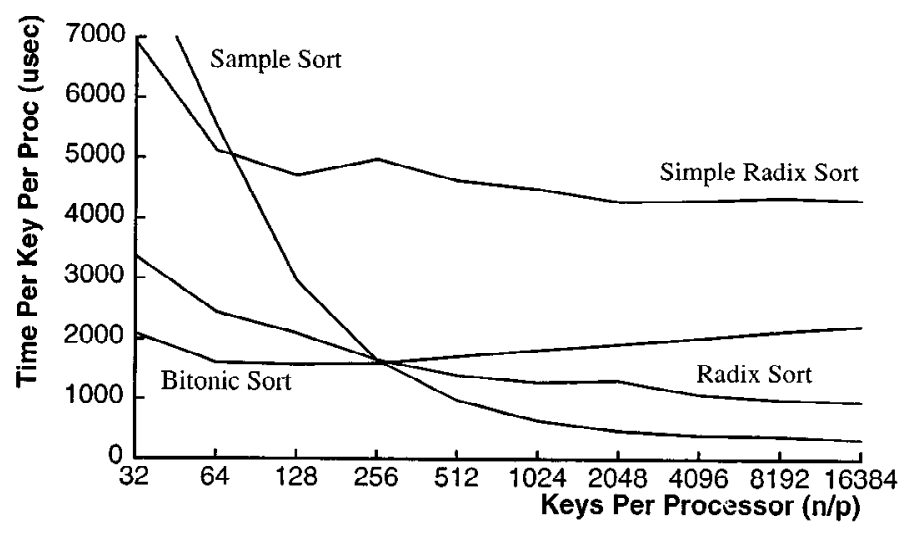

Fig. 1. Actual running times for sorting 64-bit keys on a 32K Connection Machine CM-2. In the figure the running times are divided by the number of keys per processor to permit extrapolation to machines with different numbers of processors. The term processor, as used in this paper, is a 32-bit wide so-called Sprint node, of which there are $p=1024$ in a 32K CM-2. To determine the total running time of a sort involving $n$ keys, multiply the time per key per processor in the figure by $n / p$.

bitonic sort is the slowest of the three sorts when $n / p$ is large, it is more space-efficient than the other two algorithms, and represents the fastest alternative when $n / p$ is small. Based on various pragmatic issues, the radix sort was selected to be used as the library sort for Fortran now available on the CM-2.

We have modeled the running times of our sorts using equations based on problem size, number of processors, and a set of machine parameters (e.g., time for point-topoint communication, hypercube communication, scans, and local operations). These equations serve several purposes. First, they make it easy to analyze how much time is spent in various parts of the algorithms. For example, the ratio of computation to communication for each algorithm can be quickly determined and how this is affected by problem and machine size can be seen. Second, they make it easy to generate good estimates of running times on variations of the algorithms without having to implement them. Third, it can be determined how various improvements in the architecture would improve the running times of the algorithms. For example, the equations make it easy to determine the effect of doubling the performance of message routing. Fourth, in the case of radix sort we are able to use the equations analytically to determine the best radix size as a function of the problem size. Finally, the equations allow anyone to make reasonable estimates of the running times of the algorithms on other machines. For example, the radix sort has been implemented and analyzed on the Cray Y-MP [25], and the Thinking Machines CM-5 [22], which differs significantly from the CM-2. In both cases, when appropriate values for the machine parameters are used, our equations accurately predicted the running times. Similar equations are used by Stricker [21] to analyze the running time of bitonic sort on the iWarp, and by Hightower et al. [12] to analyze the running time of flashsort on the Maspar MP-1.

The remainder of this paper studies the implementations of bitonic sort, radix sort, and sample sort. In each case it describes and analyzes the basic algorithm, as well as any 
enhancements and/or minor modifications that we introduced to optimize performance. After describing the primitive operations that our algorithms use in Section 2, Sections 3, 4 , and 5 present our studies of bitonic sort, radix sort, and sample sort, respectively. In Section 6 we compare the relative performance of these three sorts, not only in terms of running time, but also with respect to such criteria as stability and space. Appendix A presents a brief analysis of other algorithms that we considered for implementation. Appendix B presents a probabilistic analysis of the sampling procedure used in our sample sort algorithm.

\section{Primitive Operations}

This section describes a set of primitive parallel operations that can be used to implement a parallel sorting algorithm. These operations are likely to be found on any parallel computer, with the possible exception of the cube swap operation, which applies only to hypercube-based machines. All of the algorithms in this paper are described in terms of these operations. Although the costs of the operations are given only for the CM-2, our analysis can be applied to other machines by substituting the appropriate costs.

There are four classes of operations:

- Arithmetic: A local arithmetic or logical operation on each processor. Also included are global operations involving a front-end machine and the processors, such as broadcasting a word from the front end to all processors.

- Cube swap: Each processor sends and receives one message across each of the dimensions of the hypercube.

- Send: Each processor sends one message to any other processor through a routing network. In this paper we use two types of sends: a single-destination send and a send-to-queue. In the single-destination send (used in our radix-sort) messages are sent to a particular address within a particular processor, and no messages may have the same destination. In the send-to-queue (used in our sample sort) messages are sent to a particular processor and are placed in a queue in the order they are received.

- Scan: A parallel-prefix (or suffix) computation on integers, one per processor. Scans operate on a vector of input values using an associative binary operator such as integer addition. (The only operator employed by our algorithms is addition.) As output, the scan returns a vector in which each position has the "sum," according to the operator, of those input values in lesser positions. For example, a plus-scan (with integer addition as the operator) of the vector

$$
\left[\begin{array}{llllllllllll}
4 & 7 & 1 & 0 & 5 & 2 & 6 & 4 & 8 & 1 & 9 & 5
\end{array}\right]
$$

yields

$\left[\begin{array}{llllllllllll}0 & 4 & 11 & 12 & 12 & 17 & 19 & 25 & 29 & 37 & 38 & 47\end{array}\right]$

as the result of the scan.

In this paper we describe our algorithms in English, and, where more precision is required, in a parallel vector pseudocode. We generally assume that the variable $n$ refers to the number of keys to be sorted, and that $p$ is the number of processors in the machine. 
In the parallel vector pseudocode we assume that data is stored in two kinds of variables: $n$-element vectors and scalars. Vectors are identified by capitalized variable names, whereas scalar variable names are uncapitalized. Parallel arithmetic operations on vectors are performed in an elementwise fashion. The special vector Self refers to the vector of coordinate indices $(\operatorname{Self}[i]=i)$. Cube swaps along one dimension of the hypercube are performed on a vector $V$ by an operation $\operatorname{CUBE-SWAP}(V, j)$, which returns a vector whose $i$ th coordinate is $V\left[i+2^{j}\right]$ if the $j$ th bit of $i$ (in binary) is 0 , and $V\left[i-2^{j}\right]$ if the $j$ th bit of $i$ is 1 . Cube swaps along all dimensions simultaneously are described in English. A single-destination send is accomplished by the operation $\operatorname{SEND}(V, D e s t)$, which returns the vector whose $i$ th coordinate is that $V[j]$ such that $\operatorname{Dest}[j]=i$. Scan operations are performed by a procedure $\operatorname{SCAN}(V)$ that returns the plus-scan of the vector.

\subsection{Primitive Operations on the CM-2}

The CM-2 is a single-instruction multiple-data (SIMD) computer. In its full $64 \mathrm{~K}$ processor configuration, it can be viewed as $2048\left(2^{11}\right)$ Sprint nodes configured as an 11-dimensional hypercube. (A $d$-dimensional hypercube is a network with $2^{d}$ nodes in which each node has a $d$-bit label, and two nodes are neighbors if their labels differ in precisely on bit position.) The Sprint nodes have multiport capability: all dimensions of the hypercube can be used at the same time. The Sprint nodes are controlled by a front-end processor (typically a Sun 4 or Vax). Figure 2 illustrates the organization of a Sprint node, which consists of the following chips:

- Two processor chips, each containing 16 1-bit processors, a 1-bit bidirectional wire to each of up to 11 neighboring nodes in the hypercube, and hardware for routing support.

- Ten DRAM chips, containing a total of between $256 \mathrm{~K}$ bytes and $4 \mathrm{M}$ bytes of error-corrected memory, depending on the configuration. All recent machines contain at least $1 \mathrm{M}$ bytes of memory per node.

- A floating-point chip (FPU) capable of 32-bit and 64-bit floating-point arithmetic, as well as 32-bit integer arithmetic.

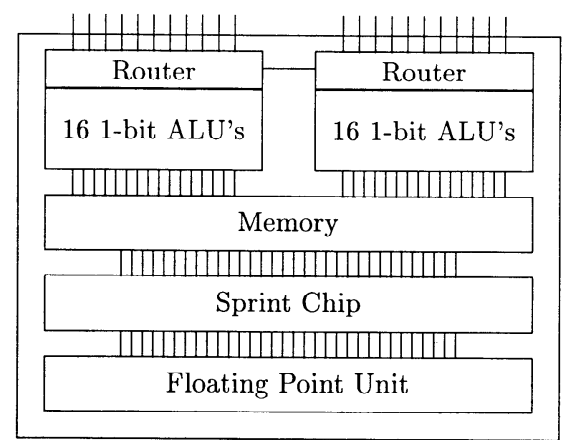

Fig. 2. The organization of a CM-2 Sprint node. 
Table 1. The time required for operations on a $32 \mathrm{~K}$ Connection Machine CM-2.*

\begin{tabular}{lcr}
\hline Operation & Symbolic time & Actual time \\
\hline Arithmetic & $A \cdot(n / p)$ & $1 \cdot(n / 1024)$ \\
Cube Swap & $Q \cdot(n / p)$ & $40 \cdot(n / 1024)$ \\
Send (routing) & $R \cdot(n / p)$ & $130 \cdot(n / 1024)$ \\
Scan (parallel prefix) & $3 A \cdot(n / p)+S$ & $3 \cdot(n / 1024)+50$ \\
\hline
\end{tabular}

* The value $p$ is the number of processors (Sprint nodes), and $n$ is the total number of elements being operated on. All operations are on 64-bit words, except for scans which are on 32-bit words. All times are in microseconds.

- A Sprint chip that serves as an interface between the memory and the floatingpoint chip. The Sprint chip contains 128 32-bit registers and has the capability to convert data from the bit-serial format used by the 1-bit processors to the 32-bit word format used by the floating-point chip.

In this paper we view each Sprint node as a single processor, rather than considering each of the $64 \mathrm{~K} 1$-bit processors on a fully configured CM-2 as separate processors. This point of view makes it easier to extrapolate our results on the CM-2 to other hypercube machines, which typically have 32- or 64-bit processors. Furthermore, it is closer to the way in which we viewed the machine when implementing the sorting algorithms. Our programs for the CM-2 were written in Connection Machine assembly language (Paris) and high-level microcode (CMIS).

Table 1 gives estimated running times for each of the four classes of primitives on a $32 \mathrm{~K} \mathrm{CM}-2$. We assume that each of $p=1024$ processors contains $n / p$ elements, for a total of $n$ elements. Times are given for 64-bit data, except for scans, which operate on 32-bit data. With respect to the operation times, we have generally simplified our expressions by ignoring fixed overheads whenever they are small, concentrating instead on throughput. (For scans, the fixed overhead is substantial, so we have included it explicitly.) Because of these simplifications, our analyses do not accurately model performance when the number of elements per processor is small. When $n / p$ is large, however, they are accurate to within approximately $10 \%$. Since most data on the CM-2 originates in the 1-bit processors, $n / p$ is typically at least 32 . As a practical matter, most sorting applications involve $n / p \geq 128$, and, often, $n / p=2048$ or much larger.

We now discuss in somewhat more detail the time estimates for each of the classes of operations.

The time $A$ for arithmetic operations is nominally chosen to be 1 microsecond. For example, the cost of summing two integer values, including the costs of loading and storing the data into local memory and of incrementing a counter (assuming the operation is in a loop) is about 1.4A. An indirect access in which different processors access potentially different memory locations requires about $3 A$ time. Also, computing the maximum (or minimum) of two values require about $3 A$ time, since these operations involve a compare followed by a conditional memory move. Throughout the paper the coefficients of $A$ were obtained empirically. For radix sort and sample sort, we instrumented the code with calls to a real-time clock which provides accurate and deterministic 
timings. The constant coefficient reported for bitonic merge was determined by fitting a curve to the timing data.

On the CM-2, the time $Q$ for cube swapping is the same whether a processor sends one message across just one dimension of the hypercube or 11 messages each across one of the 11 dimensions of the hypercube. To exploit the communication bandwidth provided by the hypercube fully, it is desirable, of course, to use all dimensions simultaneously.

The time $R$ given for a send is based on routing messages randomly, where each message is equally likely to go to any other processor. The time for the two types of sends (i.e., single-destination and send-to-queue) is approximately the same as long as in the send-to-queue the number of messages received at each processor is approximately balanced. Some variation in the time for a send occurs because some routing patterns take longer than others. As long as there is no congestion at the receiving processor, however, no known pattern takes longer than $2.5 R$. If each processor is receiving approximately the same number of messages, congestion can be avoided by injecting messages into the router in a pseudorandom order. The CM-2 also supports combining sends.

Consider the cost of a single scan operation on the CM-2 when the number of elements per processor is large. In this case the running time is only about $3 A \cdot(n / p)$, since the fixed overhead $S$ can be safely ignored. In the case of multiple independent scans (each on one element per processor), however, the fixed overhead $S$ must be taken into consideration. The other operations (Cube Swap and Send) have fixed overheads as well, but they are negligible by comparison.

\section{Batcher's Bitonic Sort}

Batcher's bitonic sort [3] is a parallel merge sort that is based upon an efficient technique for merging so-called "bitonic" sequences. A bitonic sequence is one that increases monotonically and then decreases monotonically, or can be circularly shifted to become so. One of the earliest sorts, bitonic sort was considered to be the most practical parallel sorting algorithm for many years. The theoretical running time of the sort is $\Theta\left(\lg ^{2} n\right)$, where the constant hidden by $\Theta$ is small. Moreover, bitonic sort makes use of a simple fixed communication pattern that maps directly to the edges of the hypercube; a general routing primitive need not be invoked when bitonic sort is implemented on the hypercube.

In this section we discuss our implementation of bitonic sort. The basic algorithm runs efficiently on a hypercube architecture, but uses only one dimension of the hypercube wires at a time. The CM-2 hypercube has multiport capability, however, and, by pipelining the algorithm, it is possible to make efficient use of all hypercube wires at once. This optimization results in a five-fold speedup of the communication and over a two-fold speedup in the total running time of the algorithm. Even with this optimization, the other two algorithms that we implemented outperform bitonic sort when the number $n / p$ of keys per processor is large. When $n / p$ is small, however, bitonic sort is the fastest of the three, and uses considerably less space than the other two.

Figure 3 illustrates the bitonic sort algorithm. The key step is an operation called a bitonic merge. The inputs to this operation are a pair of sequences that are sorted in opposite directions, one in ascending order and the other in descending order, so 


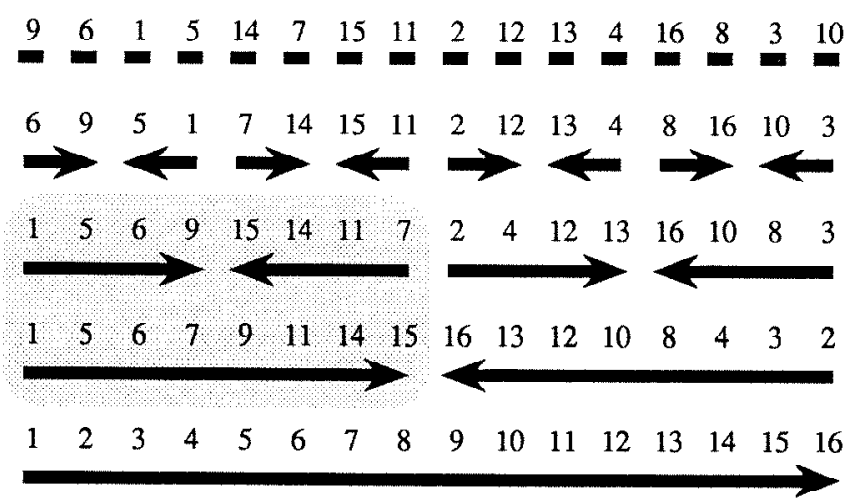

Fig. 3. An illustration of the BITONIC-SORT procedure. Each arrow represents a sequence of keys sorted in the direction of the arrow. The unsorted input sequence of $n$ keys is shown at the top, and the sorted output sequence is shown at the bottom. A bitonic merge operation is shaded. During the $d$ th step of the algorithm (where $1 \leq d \leq \lg n$ ), $n / 2^{d}$ merges are performed, each producing a sorted sequence of length $2^{d}$ from two sorted sequences of length $2^{d-1}$.

that together they form a bitonic sequence. Bitonic merge takes this bitonic sequence and from it forms a single sorted sequence. (In fact, bitonic merge will form a sorted sequence from any bitonic sequence.) For the moment, we assume that we have $n$ input keys to be sorted and that we have $p=n$ processors, each with one key. For each integer $d=1, \ldots, \lg n$, the algorithm performs $n / 2^{d}$ merges, where each merge produces a sorted sequence of length $2^{d}$ from two sorted sequences of length $2^{d-1}$.

The key step of bitonic sort is the merge operation, which is described by the following pseudocode:

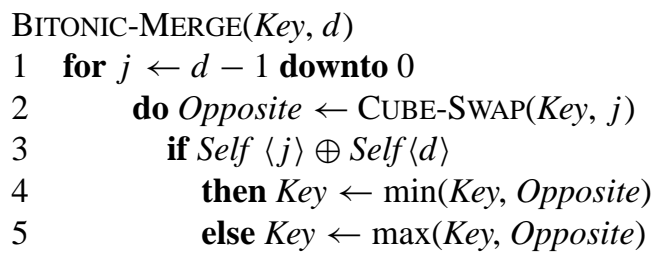

In line 3 the operator $\oplus$ denotes the exclusive-or function, and the expression "Self $\langle j\rangle$ " means the $j$ th bit of the integer representing the position of the key in the input vector. Self $\langle 0\rangle$ is the least-significant bit. Self $\langle j\rangle$ determines whether the keys should be sorted in increasing or decreasing order.

The operation of this algorithm can be understood with the help of Figure 4, which shows how two sorted sequences (i.e., a single bitonic sequence) are merged into a single ascending sequence. Each vertical line of the figure represents a processor in the hypercube, each of which initially contains one of the input keys. Time moves downward in the diagram, with the two sorted input sequences at the top, and the final single sorted sequence at the bottom. During a single step of the algorithm, all keys are communicated 


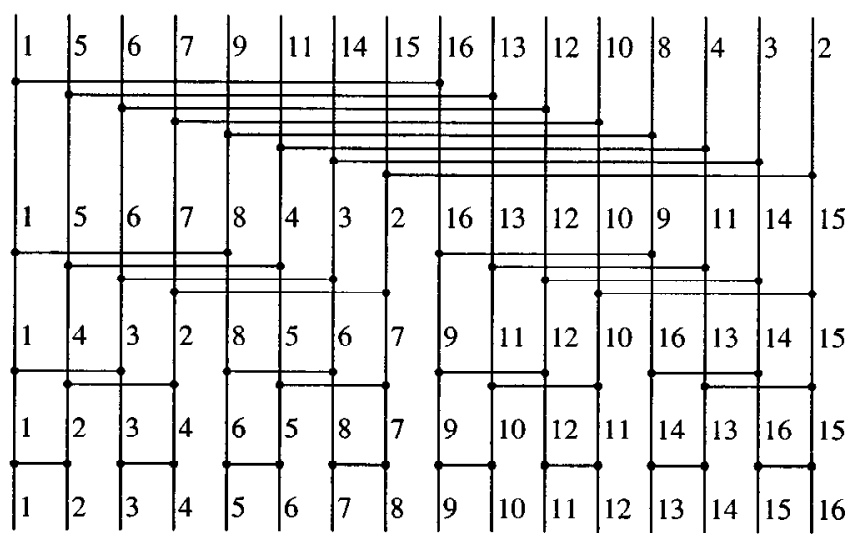

Fig. 4. Viewing the BITONIC-MERGE procedure as a hypercube algorithm. Each vertical line represents a hypercube processor. Each horizontal line segment represents the communication of keys along the hypercube wire between two processors.

across a single dimension of the hypercube. After keys have been communicated across all the dimensions of the hypercube, the hypercube processors contain the output sorted in ascending order.

Each iteration of the loop in BITONIC-MERGE is represented by the collection of horizontal line segments in a shaded region of the figure. Each horizontal line segment represents the communication of keys between two processors along a hypercube wire, which corresponds to the CUBE-SwAP in line 2. In the algorithm, Self $\langle d\rangle$ tells whether we are producing an ascending (0) or descending (1) order, and $\operatorname{Sel}\langle j\rangle$ tells whether the processor is on the left $(0)$ or right (1) side of a wire. For the example in the figure, we are sorting into ascending order $(\operatorname{Self}\langle d\rangle=0)$, and thus for each pair of keys that are swapped, the smaller replaces the key in the processor on the left and the larger is kept on the right.

We do not prove the correctness of this well-known algorithm; the interested reader is referred to [2] and [7].

To this point, we have assumed that the number $n$ of input keys is equal to the number $p$ of processors. In practice, it is important for a sorting algorithm to be able to cope with unequal values of $n$ and $p$. As it happens, the best hypercube algorithms to date use substantially different techniques for the cases $n \ll p$ and $n \gg p$. This project focuses entirely on the development of sorting algorithms for the more frequently occurring case when $n \geq p$.

To handle multiple keys per processor, we view each key address as being composed of a processor address (high-order bits corresponding to "physical" hypercube dimensions) and an index within the processor (low-order bits corresponding to "virtual" hypercube dimensions). In a bitonic merge, communication occurs across successive dimensions, in descending order. Across any physical dimension, this communication is realized by a set of $n / p$ cube swaps. After processing the physical dimensions, what remains to be performed amounts to a bitonic merge within each processor. Given $n$ keys 
and $p$ processors, the CM-2 time for the bitonic merge becomes

$$
T_{\text {merge }}= \begin{cases}(n / p) \cdot 5 A \cdot d & \text { if } d \leq \lg (n / p) \\ (n / p) \cdot(Q \cdot(d-\lg (n / p))+5 A \cdot d) & \text { if } d>\lg (n / p)\end{cases}
$$

where the coefficient 5 was determined empirically by fitting to the data. (If $d \leq \lg (n / p)$, then the bitonic merges occur entirely within processors, and so the coefficient of $Q$ is 0 .)

The bitonic sort algorithm calls the BITONIC-MERGE subroutine once for each dimension.

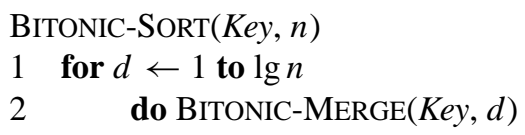

The time taken by the algorithm is

$$
\begin{aligned}
T_{\text {bitonic }} & =\sum_{d=1}^{\lg n} T_{\text {merge }} \\
& =Q \cdot(n / p)(\lg p)(\lg p+1) / 2+5 A \cdot(n / p)(\lg n)(\lg n+1) / 2 \\
& \approx 0.5 Q \cdot(n / p) \lg ^{2} p+2.5 A \cdot(n / p) \lg ^{2} n .
\end{aligned}
$$

We examine this formula more closely. The times in Table 1 indicate that $Q$ is 40 times larger than $A$, and $\lg n$ is at most two or three times larger than $\lg p$ for all but enormous volumes of data. Thus, the first term in (1), corresponding to communication time, dominates the arithmetic time for practical values of $n$ and $p$.

The problem with this naive implementation is that it is a single-port algorithm: communication occurs across only one dimension of the hypercube at a time. By using all of the dimensions virtually all of the time, we can improve the algorithm's performance significantly. The idea is to use a multiport version of BITONIC-MERGE that pipelines the keys across all dimensions of the hypercube. In the multiport version, a call of the form BitoniC-Merge $(K e y, d)$ is implemented as follows. On the first step, all processors cube swap their first keys across dimension $d$. On the second step, they cube swap their first keys across dimension $d-1$, while simultaneously cube swapping their second keys across dimension $d$. Continuing the pipelining in this manner, the total number of steps to move all the keys through $d-\lg (n / p)$ physical dimensions is $n / p+d-\lg (n / p)-1$. This algorithm is essentially equivalent to a pipelined bitonic merge on a butterfly network.

Thus, pipelining improves the time for bitonic merging to

$$
T_{\text {multiport-merge }}=\left\{\begin{array}{ll}
(n / p) \cdot 5 A \cdot d & \text { if } \quad d \leq \lg (n / p), \\
& +(n / p+d-\lg (n / p)-1)
\end{array} \quad \text { if } \quad d>\lg (n / p) .\right.
$$

By summing from $d=1$ to $\lg n$, the time for the entire multiport bitonic sort, therefore, becomes

$$
\begin{aligned}
T_{\text {multiport-bitonic }} & =Q \cdot(\lg p)\left(n / p+(\lg p) / 2-\frac{1}{2}\right)+5 A \cdot(n / p)(\lg n)(\lg n+1) / 2 \\
& \approx Q \cdot\left((n / p) \lg p+0.5 \lg ^{2} P\right)+2.5 A \cdot(n / p) \lg ^{2} n .
\end{aligned}
$$



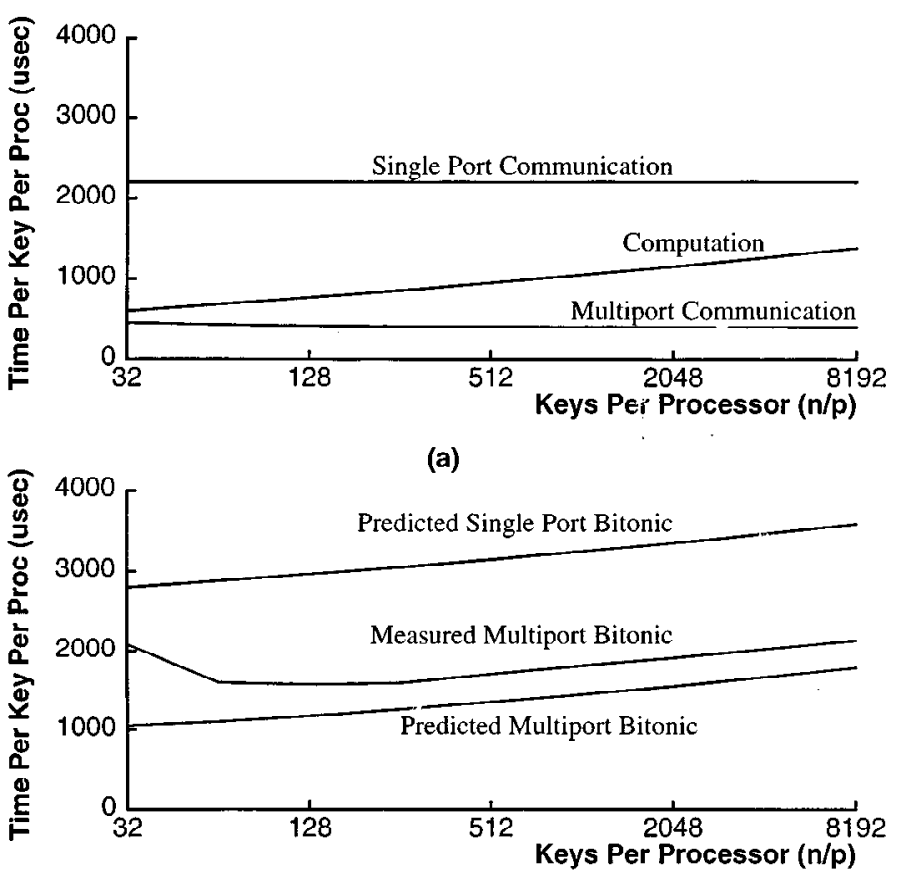

(b)

Fig. 5. Bitonic sorting 64-bit keys on a $32 \mathrm{~K} \mathrm{CM}-2(p=1024)$. (a) The predicted single-port communication is approximately five times the predicted multiport communication time. (b) The measured performance of multiport bitonic sort closely matches the predicted performance, but contains a fixed overhead.

Compare this formula with the single-port result of (1). For $n=O(p)$, the two running times do not differ by more than a constant factor. For $n=\Omega(p \lg p)$, however, the coefficient of $Q$ is $\Theta(\lg p)$ times smaller in the multiport case. Thus, total communication time is considerably reduced by pipelining when $n / p$ is large. The number of arithmetic operations is not affected by pipelining.

Figure 5(a) shows the communication and computation components of the running time for both the single-port and multiport versions of bitonic sort. These times are generated from (1) and (2). The computation component is equivalent for both algorithms. Figure 5(b) shows the predicted total time for the single-port and multiport bitonic, and the measured performance of our implementation of the multiport algorithm. The difference between predicted and measured times for small values of $n / p$ is mostly due to the fact that our equations ignore constant overhead. The difference at high $n / p$ is due to some overhead in our implementation caused by additional memory moves, effectively increasing the cost $Q$ of the cube swap. This overhead could be eliminated by an improved implementation, but the resulting algorithm would still not be competitive with sample sort for large values of $n / p$.

Multiport bitonic sort can be further improved by using a linear-time serial merge instead of a bitonic merge in order to execute the merges that occur entirely within a 
processor [4]. We estimated that the time for a processor to merge two sorted sequences of length $(n / 2 p)$ to form a single sorted sequence of length $(n / p)$ is approximately $(n / p) \cdot 10 A$. The constant is large because of the indirect addressing that would be required by the implementation. In this case the time for multiport-merge becomes

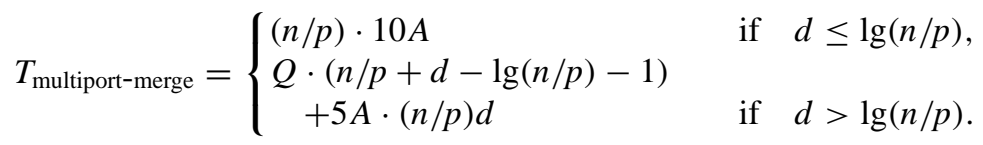

This variation yields a final running time of

$$
\begin{aligned}
T_{\text {multiport-bitonic }} \approx & Q \cdot\left((n / p) \lg p+0.5 \lg ^{2} p\right) \\
& +A \cdot(n / p)\left(2.5 \lg ^{2} p-5 \lg p+10 \lg n\right) .
\end{aligned}
$$

For large $n / p$, this formula reduces the $A$ term by a factor of 2 or more relative to (2). Once again, this improvement would not yield an algorithm that is close to the performance of the sample sort, and thus we decided not to implement it. Furthermore, the local merges could not be executed in place, so that the algorithm would lose one of its major advantages: it would no longer only require a fixed amount of additional memory.

\section{Radix Sort}

The second algorithm that we implemented is a parallel version of a counting-based radix sort [7, Section 9.3]. In contrast with bitonic sort, radix sort is not a comparison sort: it does not use comparisons alone to determine the relative ordering of keys. Instead, it relies on the representation of keys as $b$-bit integers. (Floating-point numbers can also be sorted using radix sort. With a few simple bit manipulations, floating-point keys can be converted to integer keys with the same ordering and key size. For example, IEEE double precision floating-point numbers can be sorted by inverting the mantissa and exponent bits if the sign bit is 1 , and then inverting the sign bit. The keys are then sorted as if they were integers.) Our optimized version of radix sort is quite fast, and it was the simplest to code of the three sorting algorithms that we implemented.

The basic radix sort algorithm (whether serial or parallel) examines the keys to be sorted $r$ bits at a time, starting with the least-significant block of $r$ bits in each key. Each time through the loop, it sorts the keys according to the $r$-bit block currently being considered in each key. Of fundamental importance is that this intermediate radix- $2^{r}$ sort be stable: the output ordering must preserve the input order of any two keys whose $r$-bit blocks have equal values.

The most common implementation of the intermediate radix- $2^{r}$ sort is as a counting sort. We first count to determine the rank of each key-its position in the output orderand then we permute the keys to their respective locations. The following pseudocode describes the implementation:

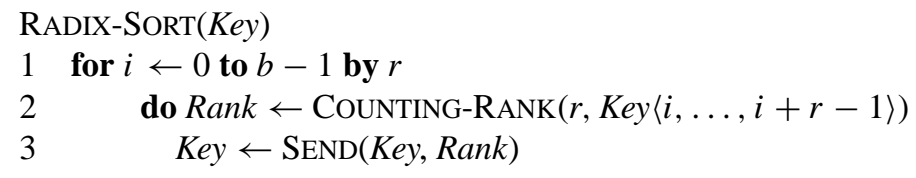


Since the algorithm requires $b / r$ passes, the total time for a parallel sort is

$$
T_{\text {radix }}=(b / r) \cdot\left(R \cdot(n / p)+T_{\text {rank }}\right),
$$

where $T_{\text {rank }}$ is the time taken by COUNTING-RANK.

The most interesting part of radix sort is the subroutine for computing ranks called in line 2. We first consider the simple algorithm underlying the original Connection Machine library sort [5], which was programmed by one of us several years ago. In the following implementation of COUNTING-RANK, the vector Block holds the $r$-bit values on which we are sorting.

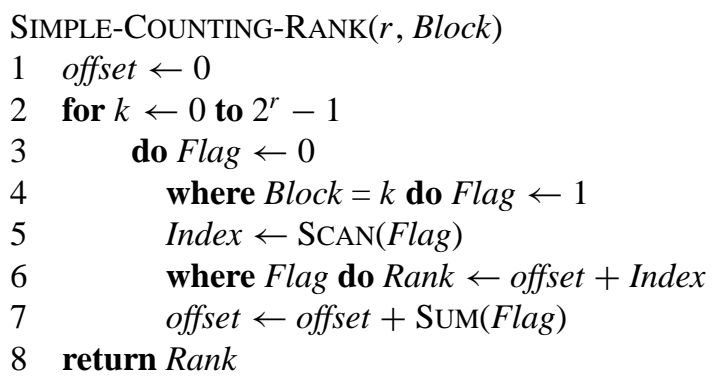

In this pseudocode the where statement executes its body only in those processors for which the condition evaluates to TRUE.

The SimPle-CounTING-RANK procedure operates as follows. Consider the $i$ th key, and assume that Block $[i]=k$. The rank of the $i$ th key is the number offset $_{k}$ of keys $j$ for which Block $[j]<k$, plus the number Index [i] of keys for which Block $[j]=k$ and $j<i$. (Here, offset $k$ is the value of offset at the beginning of the $k$ th iteration of the for loop.) The code iterates over each of the $2^{r}$ possible values that can be taken on by the $r$-bit block on which we are sorting. For each value of $k$, the algorithm uses a scan to generate the vector Index and updates the value of offset to reflect the total number of keys whose Block value is less than or equal to $k$.

To compute the running time of SIMPLE-COUNTING-RANK, we refer to the running times of the CM-2 operations in Table 1. On the CM-2, the SuM function can be computed as a by-product of the SCAN function, and thus no additional time is required to compute it. Assuming that we have $p$ processors and $n$ keys, the total time is

$$
\begin{aligned}
T_{\text {simple-rank }} & =2^{r} \cdot(3 A \cdot(n / p)+S)+2^{r}(2 A)(n / p) \\
& =A \cdot\left(5 \cdot 2^{r}(n / p)\right)+S \cdot 2^{r},
\end{aligned}
$$

where the coefficient 2 of $A$ in the last term of the first line was determined empirically by instrumenting the code. Here the term $2^{r} \cdot(3 A \cdot(n / p)+S)$ represents the time to perform $2^{r}$ scans, and the term $2^{r}(2 A)(n / p)$ represents the cost of the remaining operations, such as initializing the vector Flag and computing the vector Rank.

The total time for this version of radix sort—call it SIMPLE-RADIX-SORT-which uses SimPLE-COUNTING-RANK on $r$-bit blocks of $b$-bit keys, is therefore

$$
\begin{aligned}
T_{\text {simple-radix }} & =(b / r)\left(R \cdot(n / p)+T_{\text {simple-rank }}\right) \\
& =(b / r)\left(R \cdot(n / p)+5 A \cdot 2^{r}(n / p)+S \cdot 2^{r}\right) .
\end{aligned}
$$


(The library sort actually runs somewhat slower for small values of $n / p$, because of a large fixed overhead.) Notice from this formula that increasing $r$ reduces the number of routings proportionally, but it increases the arithmetic and scans exponentially.

We can determine the value of $r$ that minimizes $T_{\text {simple-radix }}$ by differentiating the right-hand side of (4) with respect to $r$ and setting the result equal to 0 , which yields

$$
r=\lg \left(\frac{(n / p) R}{(n / p) 5 A+S}\right)-\lg (r \ln 2-1) .
$$

For large $n / p$ (i.e., $n / p \gg(S / 5 A))$, the optimal value of $r$ is

$$
\begin{aligned}
r & \approx \lg (R / 5 A)-\lg (r \ln 2-1) \\
& \approx 3.9 .
\end{aligned}
$$

This analysis is borne out in practice by the CM-2 library sort, which runs the fastest for large $n / p$ when $r=4$.

We now consider an improved version of a parallel radix sort. The idea behind this algorithm was used by Johnsson [14]. We describe the new algorithm for counting ranks in terms of the physical processors, rather than in terms of the keys themselves. Thus, we view the length- $n$ input vector Block as a length- $p$ vector, each element of which is a length- $(n / p)$ array stored in a single processor. We also maintain a length- $p$ vector Index, each element of which is a length- $2^{r}$ array stored in a single processor. We describe the operation of the algorithm after giving the pseudocode:

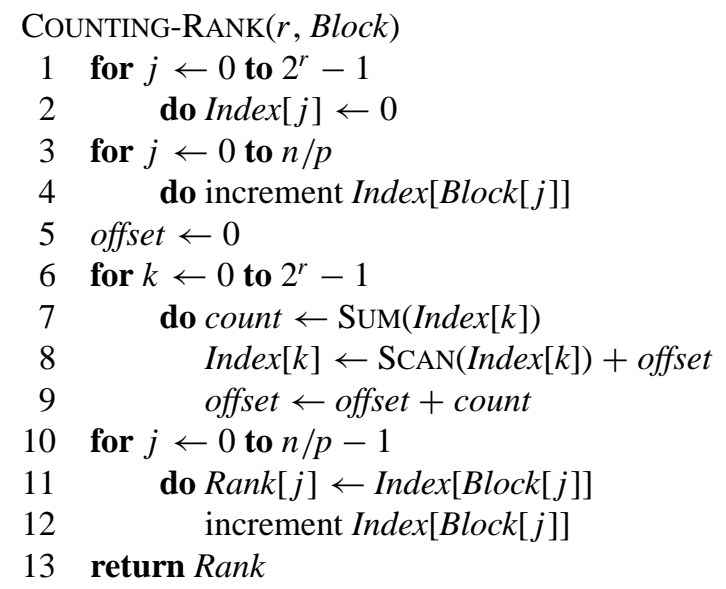

The basic idea of the algorithm is as follows. For all Block values $k=0,1, \ldots, 2^{r}-$ 1 , lines 1-4 determine how many times each value $k$ appears in each processor. Now, consider the $i$ th processor and a particular value $k$. Lines 5-9 determine the final rank of the first key, if any, in processor $i$ that has Block value $k$. The algorithm calculates this rank by computing the number offset $_{k}$ of keys with Block values less than $k$ to which it adds the number of keys with Block value equal to $k$ that are in processors 0 through $i-1$. These values are placed in the vector Index $[k]$. Having computed the overall rank of the first key in each processor (for each Block value $k$ ), the final phase of the algorithm 
(lines 10-12) computes the overall rank of every key. This algorithm requires indirect addressing, since the processors must index their local arrays independently.

The total time for COUNTING-RANK is

$$
T_{\text {rank }}=A \cdot\left(2 \cdot 2^{r}+10(n / p)\right)+S \cdot 2^{r},
$$

where the constants 2 and 10 were determined empirically. Note that $\operatorname{SUM}(\operatorname{Index}[k])$ in line 7 is actually a by-product of $\operatorname{SCAN}(\operatorname{Index}[k])$ in line 8 , and hence a single SCAN suffices. Comparing with the result obtained for SIMPLE-COUNTING-RANK, we find that the $n / p$ and $2^{r}$ terms are now additive rather than multiplicative.

The time for RADIX-SORT is

$$
\begin{aligned}
T_{\text {radix }} & =(b / r)\left(R \cdot(n / p)+T_{\text {rank }}\right) \\
& =(b / r)\left(R \cdot(n / p)+S \cdot 2^{r}+A \cdot\left(2 \cdot 2^{r}+10(n / p)\right)\right) \\
& =(b / r)\left((n / p) \cdot(R+10 A)+2^{r}(S+2 A)\right) .
\end{aligned}
$$

Figure 6 breaks down the running time of radix sort as a function of $r$ for $n / p=4096$. As can be seen from the figure, as $r$ increases, the send time diminishes and the scan time grows. We can determine the value for $r$ that minimizes the total time of the algorithm by differentiating the right-hand side of (5) with respect to $r$ and setting the result equal to 0 . For large numbers of keys per processor, the value for $r$ that we obtain satisfies

$$
\begin{aligned}
r & =\lg ((n / p)(R+10 A) /(S+2 A))-\lg (r \ln 2-1) \\
& \approx \lg (n / p)-\lg \lg (n / p)+2 .
\end{aligned}
$$

For $n / p=4096$, as in Figure 6, (7) suggests that we set $r \approx 10$, which indeed comes very close to minimizing the total running time. The marginally better value of $r=11$ can be obtained by solving (6) numerically.

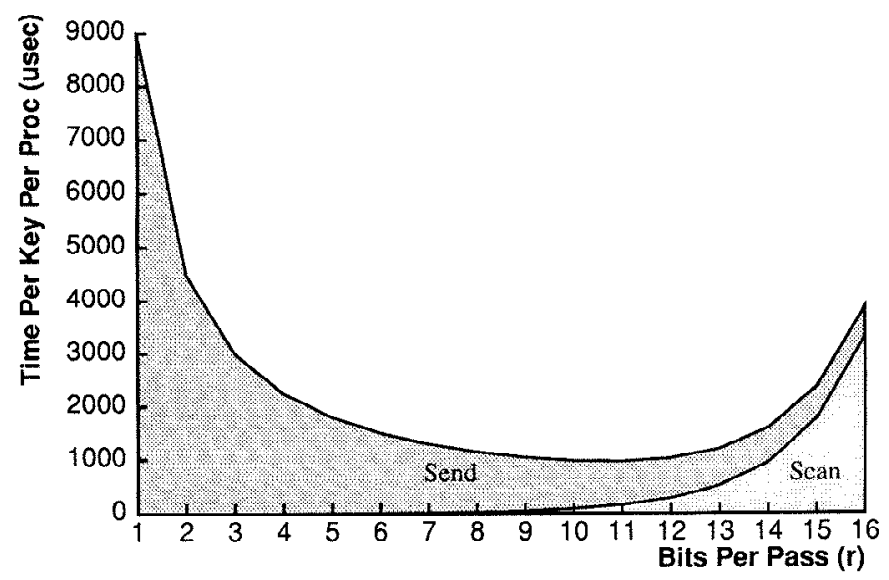

Fig. 6. A breakdown of the total predicted running time of radix sort into send time and scan time for sorting 64-bit keys $(b=64)$ with $n / p=4096$. The total running time is indicated by the top curve. The two shaded areas represent the scan time and send time. As $r$ is increased, the scan time increases and the send time decreases. (The arithmetic time is negligible.) for the parameters chosen, the optimal value of $r$ is 11 . 


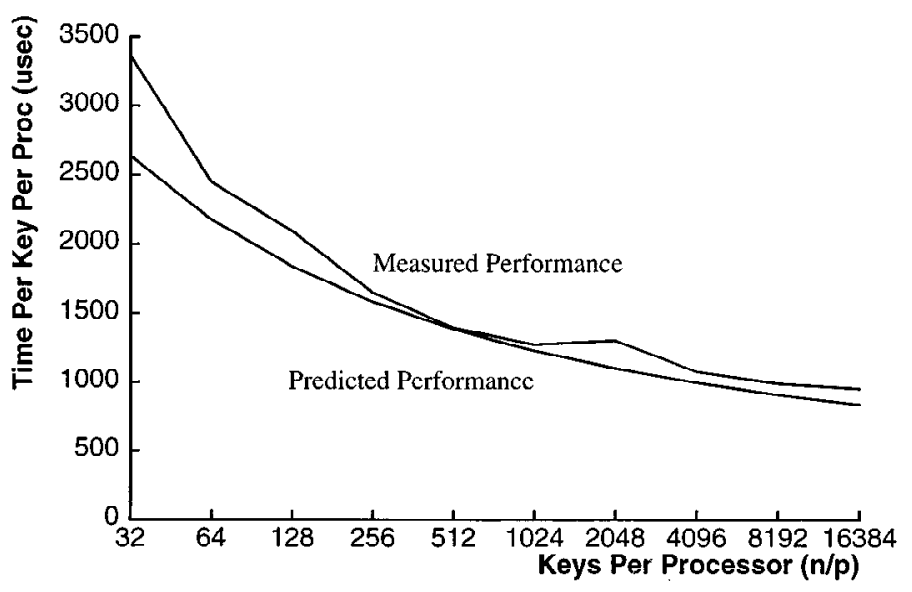

Fig. 7. Predicted and measured performance of radix sort with 64-bit keys. Measured performance is on a 32K CM-2 and uses the empirically determined optimal values for $r$. Predicted performance was calculated using (8).

Unlike the choice of $r$ dictated by the analysis of SIMPLE-RADIX-SORT, the optimal choice of $r$ for RADIX-SORT grows with $n / p$. Consequently, for large numbers of keys per processor, the number of passes of RADIX-SORT is smaller than that of SIMPLE-RADIXSORT. When we substitute our choice of $r$ back into (5), we obtain

$$
T_{\text {radix }} \approx(n / p)\left(\frac{b}{\lg (n / p)-\lg \lg (n / p)+1.5}\right)\left(R+10 A+\frac{3}{\lg (n / p)}(S+2 A)\right) .
$$

In our implementation of RADIX-SORT, the optimal values of $r$ have been determined empirically. Figure 7 compares the performance predicted by (8) with the actual running time of our implementation.

\section{Sample Sort}

The third sort that we implemented is a sample sort [10], [13], [19], [20], [24]. This sorting algorithm was the fastest for large sets of input keys, beating radix sort by more than a factor of 2. It also was the most complicated to implement. The sort is a randomized sort: it uses a random number generator. The running time is almost independent of the input distribution of keys and, with very high probability, the algorithm runs quickly.

Assuming $n$ input keys are to be sorted on a machine with $p$ processors, the algorithm proceeds in three phases:

1. A set of $p-1$ "splitter" keys are picked that partition the linear order of key values into $p$ "buckets." 
2. Based on their values, the keys are sent to the appropriate bucket, where the $i$ th bucket is stored in the $i$ th processor.

3. The keys are sorted within each bucket.

If necessary, a fourth phase can be added to load balance the keys, since the buckets do not typically have exactly equal size.

Sample sort gets its name from the way the $p-1$ splitters are selected in the first phase. From the $n$ input keys, a sample of $p s \leq n$ keys are chosen at random, where $s$ is a parameter called the oversampling ratio. This sample is sorted, and then the $p-1$ splitters are selected by taking those keys in the sample that have ranks $s, 2 s, 3 s, \ldots,(p-1) s$.

Some sample sort algorithms [10], [20], [24] choose an oversampling ratio of $s=1$, but this choice results in a relatively large deviation in the bucket sizes. By choosing a larger value, as suggested by Reif and Valiant [19] and by Huang and Chow [13], we can guarantee with high probability that no bucket contains many more keys than the average. (The Reif-Valiant flashsort algorithm differs in that it uses buckets corresponding to $O\left(\lg ^{7} p\right)$-processor subcubes of the hypercube.)

The time for Phase 3 of the algorithm depends on the maximum number, call it $L$, of keys in a single bucket. Since the average bucket size is $n / p$, the efficiency by which a given oversampling ratio $s$ maintains small bucket sizes can be measured as the ratio $L /(n / p)$, which is referred to as the bucket expansion. The bucket expansion gives the ratio of the maximum bucket size to the average bucket size. The expected value of the bucket expansion depends on the oversampling ratio $s$ and on the total number $n$ of keys, and is denoted by $\beta(s, n)$.

It is extremely unlikely that the bucket expansion will be significantly greater than its expected value. If the oversampling ratio is $s$, then the probability that the bucket expansion is greater than some factor $\alpha \leq 1+1 / s$ is

$$
\operatorname{Pr}[\beta(s, n)>\alpha] \leq n e^{-(1-1 / \alpha)^{2} \alpha s / 2} .
$$

This bound, which is proved in Appendix B, is graphed in Figure 8. As an example, with an oversampling ratio of $s=64$ and $n=10^{6}$ keys, the probability that the largest bucket is more than 2.5 times as large as the average bucket is less than $10^{-6}$.

We shall see shortly that the running time of sample sort depends linearly on both the oversampling ratio and the bucket expansion. As is apparent from Figure 8, as the oversampling ratio $s$ increases, the bucket expansion decreases. Thus, the oversampling ratio $s$ must be carefully adjusted in order to obtain optimal performance.

We are now ready to discuss our implementation of the sample sort algorithm. Before executing Phase 1, however, the algorithm must do a little preprocessing. The reason is that the basic sample sort algorithm assumes that all input keys are distinct. If many keys happen to have the same value, failure to break ties consistently between them can result in an uneven distribution of keys to buckets. Consequently, before the first phase of the sample sort begins, we tag each key with its address, thereby guaranteeing that the tagged keys all have distinct values.

Phase 1: Selecting the Splitters. The first phase of sample sort begins with each processor randomly selecting a set of $s$ tagged keys from among those stored in its local memory. We call these keys the candidates. We implement this method by partitioning 


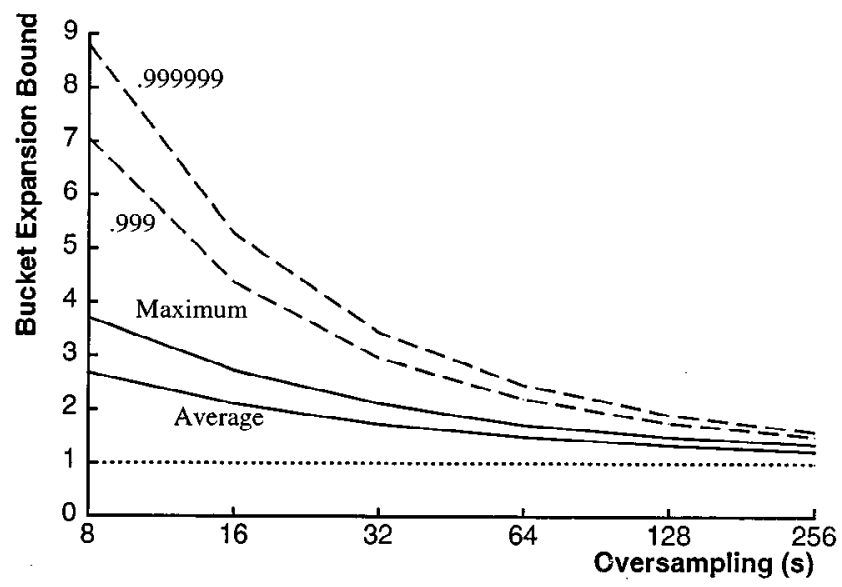

Fig. 8. Bucket expansion for sample sorting $n=10^{6}$ keys, as a function of oversampling ratio $s(p=1024)$. The dashed curves are theoretical upper bounds given by (9) when setting the probability of being within the bound to $1-10^{-3}$ (the lower dashed curve) and $1-10^{-6}$ (the upper dashed curve). The solid curves are experimental values for bucket expansion. The upper solid curve shows the maximum bucket expansion found over $10^{3}$ trials, and the lower solid curve shows the average bucket expansion over $10^{3}$ trials. In practice, oversampling ratios of $s=32$ or $s=64$ yield bucket expansions of less than 2 .

each processor's $n / p$ keys into $s$ blocks of $n / p s$ keys, and then we choose one key at random from each block. This selection process differs from that where each processor selects $s$ tagged keys randomly from the entire set, as is done in both the Reif-Valiant [19] and Huang-Chow [13] algorithms. All of these methods yield small bucket expansions. Since the CM-2 is a distributed-memory machine, however, the local-choice method has an advantage in performance over global-choice methods: no global communication is required to select the candidates. In our implementation we typically pick $s=32$ or $s=64$, depending on the number of keys per processor in the input.

Once the candidates have been selected, they are sorted across the machine using the simple version of radix sort described in Section 4. (Since radix sort is stable, the tags need not be sorted, although they must remain attached to the corresponding keys.) Since the sample contains many fewer keys than does the input, this step runs significantly faster than sorting all of the keys with radix sort. The splitters are now chosen as the keys with ranks $s, 2 s, 3 s, \ldots,(p-1) s$. The actual extraction of the splitters from the sample is implemented as part of Phase 2 .

The dominant time required by Phase 1 is the time for sorting the candidates:

$T_{\text {candidates }}=R S(p s, p)$,

where $R S(p s, p)$ is the time required to radix sort $p s$ keys on $p$ processors. Using the radix sort from the original CM-2 Paris library, we have $T_{\text {candidates }} \approx 7000 \mathrm{~A} \cdot s$.

Notice that the time for Phase 1 is independent of the total number $n$ of keys, since during the selection process, a processor need not look at all of its $n / p$ keys in order to select from them randomly. Notice also that if we had implemented a global-choice sampling strategy, we would have had a term containing $R \cdot s$ in the expression. 
Phase 2: Distributing the Keys to Buckets. Except for our local-choice method of picking a sample and the choice of algorithm used to sort the oversampled keys, Phase 1 follows both the Reif-Valiant and Huang-Chow algorithms. In Phase 2, however, we follow Huang-Chow more closely.

Each key can determine the bucket to which it belongs by performing a binary search of the sorted array of splitters. We implemented this part of the phase in a straightforward fashion: the front end reads the splitters one by one and broadcasts them to each processor. Then each processor determines the bucket for each of its keys by performing a binary search of the array of splitters stored separately in each processor. Once we have determined to which bucket a key belongs, we throw away the tagging information used to make each key unique and route the keys directly to their appropriate buckets. We allocate enough memory for the buckets to guarantee a very high probability of accommodating the maximum bucket size. In the unlikely event of a bucket overflow, excess keys are discarded during the route and the algorithm is restarted with a new random seed.

The time required by Phase 2 can be separated into the time for the broadcast, the time for the binary search, and the time for the send:

$$
\begin{aligned}
& T_{\text {broadcast }}=50 A \cdot p, \\
& T_{\text {bin-search }}=6.5 A \cdot(n / p) \lg p, \\
& T_{\text {send }}=R \cdot(n / p) ;
\end{aligned}
$$

where the constants 50 and 6.5 were determined empirically by instrumenting the code.

As is evident by our description and also by inspection of the formula for $T_{\text {broadcast }}$, the reading and broadcasting of splitters by the front end is a serial bottleneck for the algorithm. Our sample sort is really only a reasonable sort when $n / p$ is large, however. In particular, the costs due to binary search outweigh the costs due to reading and broadcasting the splitters when $6.5(n / p) \lg p>50 p$, or, equivalently, when $n / p>$ $(50 / 6.5) p / \lg p$. For a $64 \mathrm{~K} \mathrm{CM}-2$, we have $p=2048$, and the preceding inequality holds when the number $n / p$ of input keys per processor is at least 1432. This number is not particularly large, since each processor on the CM- 2 has a full megabyte of memory even when the machine is configured with only 1-megabit DRAMs.

Phase 3: Sorting Keys Within Processors. The third phase sorts the keys locally within each bucket. The time taken by this phase is equal to the time taken by the processor with the most keys in its bucket. If the expected bucket expansion is $\beta(s, n)$, the expected size of the largest bucket is $(n / p) \beta(s, n)$.

We use a standard serial radix sort in which each pass is implemented using several passes of a counting sort (see, for example, Section 9.3 of [7]). Radix sort was used because it is significantly faster than comparison sorts such as quicksort. The serial radix sort requires time

$$
T_{\text {local-sort }}=(b / r) A \cdot\left((1.3) 2^{r}+10(n / p) \beta(s, n)\right),
$$

where $b$ is the number of bits in a key and $2^{r}$ is the radix of the sort. The first term in the coefficient of $A$ corresponds to the $b / r$ (serial) scan computations on a histogram of 


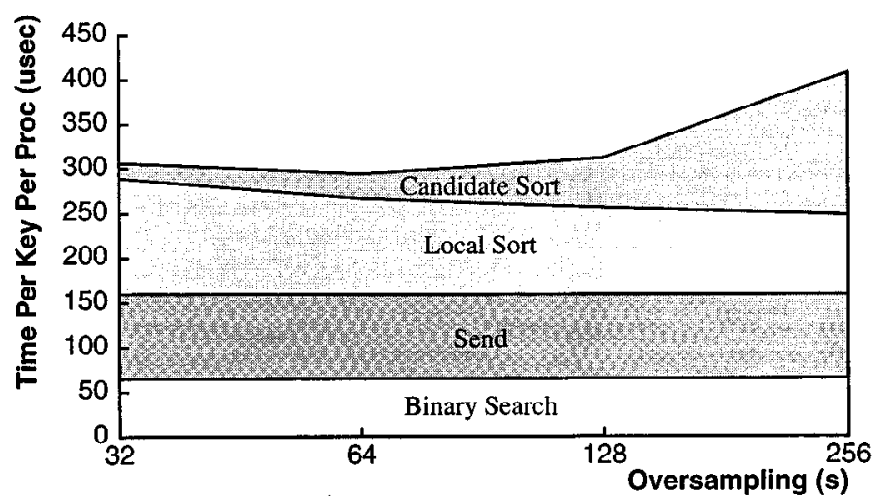

Fig. 9. Breakdown of sample sort for various choices of the oversampling ratio $s$. The graph shows the measured running time for sorting 64-bit keys $(b=64)$ with $n / p=16,384$ keys per processor on a $32 \mathrm{~K} \mathrm{CM}-2$ ( $p=1024)$, where the height of each labeled region indicates the time for the corresponding component of the sort. As the oversampling ratio is increased, two effects may be observed: (i) the time for the candidate sort increases because there are more candidates to sort, and (ii) the time for the local sort decreases because the maximum bucket expansion diminishes. For these parameters, the total time is minimized at $s=64$. $\left(T_{\text {broadcast }}\right.$ is not shown, since it is negligible.)

key values, and the second term corresponds to the work needed to count the number of keys with each $r$-bit value and to put the keys in their final destinations.

We can determine the value of $r$ that minimizes $T_{\text {local-sort }}$ by differentiating the right-hand side of (11) with respect to $r$ and setting the result equal to 0 . This yields $r \approx \lg (n / p)-1$ for large $n / p$. With this selection of $r$, the cost of the first term in the equation is small relative to the second term. Typically, $b / r \approx 6$ and $\beta(s, n) \approx 2$, which yields

$$
\begin{aligned}
T_{\text {local-sort }} & \approx 6 A \cdot 10(n / p) \cdot 2 \\
& =120 A \cdot(n / p) .
\end{aligned}
$$

Discussion. The main parameter to choose in the algorithm is the oversampling ratio $s$. A larger $s$ distributes the keys more evenly within the buckets, thereby speeding up Phase 3 of the algorithm. A larger $s$ also means a larger sample to be sorted, however, thereby causing Phase 1 of the algorithm to take longer. Figure 9 shows the tradeoff we obtained experimentally, for $n / p=16,384$. As can be seen from the figure, choosing $s=64$ is optimal in this case.

To obtain the arithmetic expression that describes the total running time of the sample sort, we sum the formulas for the phases, which results in the expression

$$
\begin{aligned}
T_{\text {sample }} & =T_{\text {candidates }}+T_{\text {broadcast }}+T_{\text {bin-search }}+T_{\text {send }}+T_{\text {local-sort }} \\
& \approx 7000 A \cdot s+50 A \cdot p+6.5 A \cdot(n / p) \lg p+R \cdot(n / p)+120 A \cdot(n / p),
\end{aligned}
$$

where we have dropped inessential terms. Figure 10 shows the experimental breakdown of times for the various tasks accomplished by the algorithm, which closely match this equation. 


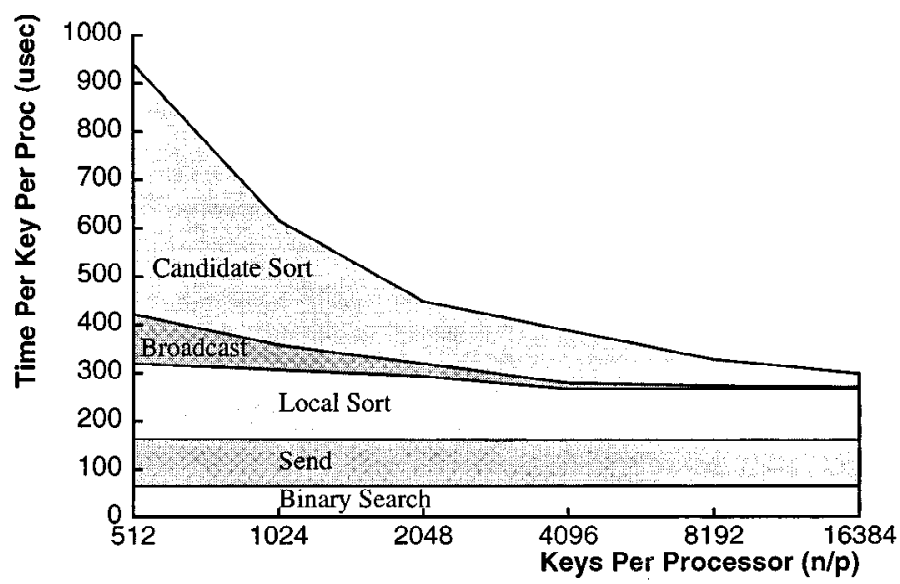

Fig. 10. A breakdown of the actual running time of sample sort, as a function of the input size $n$. The graph shows actual running times for 64-bit keys on a 32K CM-2 $(p=1024)$. The per-key cost of broadcasting splitters decreases as $n / p$ increases, since the total cost of the broadcast is independent of $n$. The per-key cost of the candidate sort decreases until there are $4 \mathrm{~K}$ keys per processor; at this point, we increase the oversampling ratio from $s=32$ to $s=64$ in order to reduce the time for local sorting. The local sort improves slightly at higher values of $n / p$, because the bucket expansion decreases while the per-key times for send and binary search remain constant.

We look more closely at the formula for $T_{\text {sample }}$ as $n / p$ becomes large. The first two terms, which correspond to the sorting of candidates and the broadcasting of splitters, become insignificant. On a 64K CM-2, the other three terms grow proportionally. Specifically, the last two terms, which correspond to the send and local sort, take about the same time, and the third term, binary searching, takes about half that time. Thus, as $n / p$ becomes large, the entire algorithm runs in less than three times the cost of a single send. Since $T_{\text {sample }}$ is so close to the time of a single send, it is unlikely that any other sorting algorithm on the CM-2 can outperform it by much.

There were many variations of the sample sort algorithm that we considered implementing. We now discuss a few.

Splitter-Directed Routing. Rather than broadcasting the splitters, binary searching, and sending in Phase 2, we might have used the "splitter-directed routing" method from Reif and Valiant's flashsort. The idea is to send each key through the hypercube to its destination bucket, using the dimensions of the hypercube in some fixed order. At each hypercube node, the key chooses either to leave the node or not based on a comparison of the key to a splitter value stored at the node. Each key therefore follows a path through the network to its bucket based on $\lg p$ comparisons, one for each dimension of the network. On the CM-2, the algorithm can be pipelined across the cube wires in a way that is similar to the pipelined version of bitonic sort. The local processing required at each step of the routing is quite involved, however. It requires managing queues since a variable number of messages can arrive at each node. Our analysis indicated that although communication costs for splitter-directed routing might take less time 
than the communication costs required to simply route messages through the network, this advantage could not be exploited, because bookkeeping (arithmetic) costs would dominate. Our conclusion was that splitter-directed routing may be a reasonable option if it is supported by special-purpose hardware. Lacking that on the CM-2, the scheme that we chose to implement was faster and much simpler to code. Since our original work, Hightower et al. have implemented a version of splitter-directed routing [12] on a toroidal mesh and found that when the number of keys per processor is not large, splitter directed routing can outperform sample sort on the Maspar MP-1.

Smaller Sets of Keys. As implemented, sample sort is suitable only when the number $n / p$ of keys per processor is large, especially since we broadcast the $p-1$ splitters from the front end. One way to improve the algorithm when each processor contains relatively few input keys would be to execute two passes of Phases 1 and 2. In the first pass we can generate $\sqrt{p}-1$ splitters and assign a group of $\sqrt{p}$ processors to each bucket. Each key can then be sent to a random processor within the processor group corresponding to its bucket. In the second pass each group generates $\sqrt{p}$ splitters which are locally broadcast within the subcubes, and then keys are sent to their final destinations. With this algorithm, many fewer splitters need to be distributed to each processor, but twice the number of sends are required. This variation was not implemented, because we felt that it would not outperform bitonic sort for small values of $n / p$.

Load Balancing. When the three phases of the algorithm are complete, not all processors have the same number of keys. Although some applications of sorting — such as implementing a combining send or heuristic clustering-do not require that the processor loads be exactly balanced, many do. After sorting, load balancing can be performed by first scanning to determine the destination of each sorted key and then routing the keys to their final destinations. The dominant cost in load balancing is the extra send. We implemented a version of sample sort with load balancing. With large numbers of keys per processor, the additional cost was only $30 \%$, and the algorithm still outperforms the other sorts.

Key Distribution. The randomized sample sort algorithm is insensitive to the distribution of keys, but, unfortunately, the CM-2 message router is not, as was mentioned in Section 2. In fact, for certain patterns, routing can take up to two and a half times longer than normally expected. This difficulty can be overcome, however, by randomizing the location of buckets. For algorithms that require the output keys in the canonical order of processors, an extra send is required, as well as a small amount of additional routing so that the scan for load balancing is performed in the canonical order. This same send can also be used for load balancing.

\section{Conclusions}

Our goal in this project was to develop a system sort for the Connection Machine. Because of this goal, raw speed was not our only concern. Other issues included space, stability, portability, and simplicity. Radix sort has several notable advantages with respect to 
Table 2. Summary of the three sorting algorithms assuming 64-bit keys.*

\begin{tabular}{|c|c|c|c|c|c|c|}
\hline \multirow[b]{2}{*}{ Algorithm } & \multirow[b]{2}{*}{ Stable } & \multirow[b]{2}{*}{ Load balanced } & \multicolumn{2}{|c|}{ Time $/(n / p)$} & \multirow[b]{2}{*}{ Memory } & \multirow[b]{2}{*}{ Rank } \\
\hline & & & $n / p=64$ & $n / p=16 K$ & & \\
\hline Bitonic & No & Yes & $1600 \mu \mathrm{s}$ & $2200 \mu \mathrm{s}$ & 1.0 & 1.5 \\
\hline Radix & Yes & Yes & $2400 \mu \mathrm{s}$ & $950 \mu \mathrm{s}$ & 2.1 & 1.0 \\
\hline Sample & No & No & $5500 \mu \mathrm{s}$ & $330 \mu \mathrm{s}$ & 3.2 & 1.5 \\
\hline
\end{tabular}

*The loaded balanced column specifies whether the final result is balanced across the processors. The time column is the time to sort on a 1024-processor machine (32K CM-2). The memory column is the ratio, for large $n / p$, of the space taken by the algorithm to the space taken by the original data. The rank column is an approximate ratio of the time of a rank to the time of a sort. The rank operation returns to each key the rank it would attain if the vector were sorted.

these criteria. Radix sort is stable, easy to code and maintain, performs reasonably well over the entire range of $n / p$, requires less memory than sample sort, and performs well on short keys. Although the other two sorts have domains of applicability, we concluded that the radix sort was most suitable as a system sort.

Table 2 compares the three sorting algorithms. In the following paragraphs we examine some of the quantitative differences between the algorithms.

Running Time. A graph of the actual running times of all three sorts along with the time of the original system sort was given in Figure 1. With many keys per processor, the sample sort is approximately three times faster than the other two sorts and therefore, based on pure performance, sample sort is the clear winner.

More informative than the raw running times are the equations for the running times, since they show how the running time is affected by the number of keys, the number of processors, and various machine parameters. If we assume that $n / p$ is large, we can approximate the equations for the three algorithms as

$$
\begin{aligned}
& T_{\text {bitonic }} \approx(n / p)\left(Q \cdot(\lg p)+A \cdot 2.5\left(\lg ^{2} n\right)\right), \\
& T_{\text {radix }} \approx(n / p)(R \cdot 6+A \cdot 80), \\
& T_{\text {sample }} \approx(n / p)(R+A \cdot(5 \lg p+120)) .
\end{aligned}
$$

If $Q, R$, and $A$ are known, these equations can be used to give rough estimates of running times for the algorithms on other machines. We caution, however, that running times predicted in this fashion could err by as much as a factor of 2 . The $A$ terms in the equations are likely to be the least accurate since the constants were all derived empirically for the CM-2, and they depend highly on the local capabilities of the processors.

The equations can also give an idea of how much would be gained in each sorting algorithm by improving various aspects of the CM-2. For example, we could analyze the effect of improving the time for a send. Based on the equations, we see that radix sort would benefit the most, since its running time is dominated by the send (currently on the CM-2, $R=130 A$ ).

Space. A second important concern is the space required by each sorting algorithm. 
Bitonic sort executes in place and therefore requires only a small constant amount of additional memory per processor for storing certain temporary variables. Our radix sort, using $n$ keys, each consisting of $w$ 32-bit words, requires $2 w(n / p)+2^{r}$ 32-bit words of space per processor. The first term is needed for storing the keys before and after the send (the send cannot be executed in place), and the second term is needed for holding the bucket sums. Because of the first term, the space required by the sort is at least twice that required by the original data. The number in Table 2 corresponds to the case $w=2$ (64-bits) and $r=\lg (n / p)-2$ (set to minimize the running time). Our sample sort requires a maximum of $2 w(n / p) \beta(s, n)+2^{r}+(w+1)(p-1) 32$-bit words of space in any processor. The first and second terms are needed for local radix sorting, and the third term is needed for storing the splitters within each processor. The number in Table 2 corresponds to the case $w=2, r=\lg (n / p)-1$ (set to minimize the running time), and $\beta(s, n) \approx 1.5$ (determined from experimental values).

Ranking. Often, in practice, a "rank" is a more useful operation than a sort. For a vector of keys, the rank operation returns to each key the rank it would attain if the vector were sorted. This operation allows the user to rank the keys and then send a much larger block of auxiliary information associated with each key to the final sorted position. For each of the three algorithms that we implemented, we also implemented a version that generates the ranks instead of the final sorted order. To implement a rank operation in terms of a sort, the original index in the vector is tagged onto each key and is then carried around during the sort. Once sorted, the final index is sent back to the location specified by the tag (the key's original position). In a radix-sort-based implementation of the rank operation, the cost of the additional send can be avoided by omitting the last send of radix sort, and sending the rank directly back to the index specified by the tag. Furthermore, as each block of the key is used by radix sort, that block can be thrown away, thereby shortening the message length of subsequent sends. Because of this, the time of "radix-rank" is only marginally more expensive than that of radix sort. For sample sort and bitonic sort, carrying the tag around slows down the algorithm by a factor of between 1.3 and 1.5 .

Stability. Radix sort is stable, but the other two sorts are not. Bitonic sort and sample sort can be made stable by tagging each key with its initial index, as is done for the rank. In this case, however, not only must the tag be carried around during the sends, it must also be used in the comparisons. Sorting the extra tag can cause a slowdown of up to a factor of 1.5 .

Key Length. Another issue is sorting short keys-keys with perhaps 10, 16, or 24 significant bits. Sorting short keys is a problem that arises reasonably often in CM-2 applications. For short keys, the time required by bitonic sort is not at all improved over the 32-bit time. The time required by the sample sort is marginally improved, since the cost of the local radix sort is reduced. The time required by radix sort, however, is essentially proportional to the key length. Since $r$ is typically in the range $10 \leq r<16$, sorting 20 bits requires two passes instead of three to four for 32 bits and five to seven for 64 bits. 


\section{Acknowledgments}

Many people were involved in this project, and we would like to thank all of them. We would particularly like to thank Mark Bromley, Steve Heller, Bradley Kuszmaul, and Kevin Oliveau of Thinking Machines Corporation for helping with various aspects of the CM-2, including the implementation of the send that was needed for the sample sort. We would also like to thank John Mucci of Thinking Machines and Rita of Rita's for his support and her inspiration.

\section{Appendix A. Other Sorts of Sorts}

Many algorithms have been developed for sorting on the hypercube and related networks such as the butterfly, cube-connected cycles, and shuffle-exchange. We considered a number of these algorithms before deciding to implement bitonic sort, radix sort, and sample sort. The purpose of this section is to discuss some of the other sorting algorithms considered and, in particular, to indicate why these alternatives were not selected for implementation.

Quicksort. It is relatively easy to implement a parallel version of quicksort on the CM-2 using segmented scans. First, a pivot is chosen at random and broadcast using scans. The pivot partitions the keys into small keys and large keys. Next, using scans, each small key is labeled with the number of small keys that precede it in the linear order, and each large key is labeled with the number of large keys that precede it, plus the total number of small keys. The keys are then routed to the locations specified by their labels. The new linear order is broken into two segments, the small keys and the large keys, and the algorithm is recursively applied to each segment. The expected number of levels of recursion is close to $\lg n$, and, at each level, the algorithm performs one route and approximately seven scans. This algorithm has been implemented in a high-level language ( ${ }^{*}$ Lisp) and runs about half as fast as the original system sort. We believed that we could not speed it up significantly, since the scan and route operations are already performed in hardware.

Hyperquicksort. The hyperquicksort algorithm [23] can be outlined as follows. First, each hypercube node sorts its $n / p$ keys locally. Then one of the hypercube nodes broadcasts its median key, $m$, to all of the other nodes. This key is used as a pivot. Each node partitions its keys into those smaller than $m$, and those larger. Next, the hypercube nodes exchange keys along the dimension- 0 edges of the hypercube. A node whose address begins with 0 sends all of its keys that are larger than $m$ to its neighbor whose address begins with 1 . The neighbor sends back all of its keys that are smaller than $m$. As keys arrive at a node, they are merged into the sorted sequence of keys that were not sent by that node. Finally, the algorithm is recursively applied to the $p / 2$-node subcubes whose addresses begin with 0 and 1 , respectively.

The communication cost of hyperquicksort is comparable to that of the fully pipelined version of bitonic sort. The expected cost is at least $Q n \lg p / 2 p$ since the algorithm uses the $\lg p$ dimensions one at a time and, for each dimension, every node expects to send half of its $n / p$ keys to its neighbor. The cost of bitonic sort is always $Q \cdot(\lg p)(n / p+$ $\left.(\lg p) / 2-\frac{1}{2}\right)$ (see Section 3). 
The main advantage of bitonic sort over hyperquicksort is that its performance is not affected by the initial distribution of the keys to be sorted. Hyperquicksort relies on a random initial distribution to ensure that the work each processor has to do is reasonably balanced. Although hyperquicksort may perform less arithmetic than bitonic sort in the best case, it uses indirect addressing, which is relatively expensive on the CM-2.

Sparse Enumeration Sort. The Nassimi-Sahni sorting algorithm [17], which is referred to as sparse enumeration sort, is used when the number $n$ of items to be sorted is smaller than the number $p$ of processors. In the special case $n=\sqrt{p}$, sparse enumeration sort is a very simple algorithm indeed. The $n$ records are initially stored one-per-processor in the $n$ lowest-numbered processors; viewing the processors of the hypercube as forming a two-dimensional $n \times n$ array, the input records occupy the first row of the array. Sparse enumeration sort proceeds by performing a set of $n$ parallel column broadcasts (from the topmost entry in each column) followed by $n$ parallel row broadcasts (from the diagonal position), so that the processor at row $i$ and column $j$ of the array contains a copy of the $i$ th and $j$ th items. At this point, all pairs of items can be simultaneously compared in constant time, and prefix operations over the rows can be used to compute the overall rank of each item. The $i$ th row is then used to route a copy of item $i$ to the column corresponding to its output rank. Finally, a set of $n$ parallel column routes is used to move each item to its sorted output position in the first row. For values of $n$ strictly less than $\sqrt{p}$, sparse enumeration sort proceeds in exactly the same fashion: $n^{2}$ processors are used, and the remaining $p-n^{2}$ processors are idle. Thus, sparse enumeration sort runs in $O(\lg n)$ time when $n \leq \sqrt{p}$.

Spares enumeration sort generalizes the preceding algorithm in an elegant manner to obtain a smooth tradeoff between $O(\lg n)$ performance at $n=\sqrt{p}$ and $O\left(\lg ^{2} n\right)$ performance at $n=p$ (the performance of bitonic sort). In this range, sparse enumeration sort is structured as a $(p / n)$-way merge sort. After the $i$ th set of parallel merges, the $n$ items are organized into $n(n / p)^{i}$ sorted lists of length $(p / n)^{i}$. (Initially there are $n$ lists of length 1.) The $i$ th set of merges is performed in $O(i \lg (p / n))$ time using a constant number of bitonic merges, prefix operations, and monotone routes. Monotone routes are a special class of routing operations that can be performed deterministically on-line in a collision-free manner. On the CM-2, monotone routes would be implemented using cube swaps; the entire implementation of sparse enumeration sort would not make use of the $\mathrm{CM}-2$ router. A straightforward computation shows that the overall time complexity of sparse enumeration sort is $O\left(\lg ^{2} n / \lg (p / n)\right)$ time.

For sufficiently large values of the ratio $p / n>1$, it would be expected that sparse enumeration sort would perform better than the other sorts we looked at. It is unclear, however, that a parallel computer would be required to solve such small problems and better times might be achieved by solving the problem on a single processor, or by reducing $p$.

Column Sort. Leighton's column sort [15] is an elegant parallel sorting technique that has found many theoretical applications. Column sort sorts $n$ keys using two primitive operations. The first primitive operation is to sort $n^{1 / 3}$ separate sets (called columns) of $n^{2 / 3}$ keys each. Depending on the particular application, this sorting primitive may either be accomplished by a recursive call or, more typically, by some other sorting algorithm. 
The second primitive operation is to route all $n$ keys according to a fixed permutation. Alternating between sorts and routes four times suffices to sort all $n$ elements.

If $n \geq p^{3}$, then column sort runs quite efficiently. The sorting primitive is executed as a local sort, and all of the fixed permutations required by column sort are straightforward to implement in a greedy, collision-free manner. In terms of the CM-2, they can be implemented with a simple sequence of cube swaps rather than by invoking the router. As another implementation optimization, we remark that the "standard" column sort algorithm is not pipelined and would only make use of a $1 / \lg p$ fraction of the CM-2 wires at any give time. $\mathrm{A} \Theta(\lg p)$ speedup can be achieved by pipelining, and there are at least two approaches worthy of consideration. The first approach is to partition the data at each processor into $\lg p$ equal-sized sets, interleave $\lg p$ column sorts, and then merge the resulting $\lg p$ sorted lists. The second approach is to pipeline each of the routing operations in a single application of column sort.

The main drawback of column sort is that, for $n<p^{3}$, some degree (depending on the ratio $n / p$ ) of recursion is necessary in order to perform the sorting primitive; sets of $n^{2 / 3}$ items occupy more than a single processor. We chose not to implement column sort because it appeared that the condition $n \geq p^{3}$ would not be satisfied in many cases of interest, and a close analysis of critical sections of the potential code indicated that a recursive version of column sort would provide little, if any, improvement over either radix sort or sample sort. Furthermore, the relative performance of column sort would tend to degrade quite severely for small values of the ratio $n / p$.

The asymptotic performance of column sort is best understood by considering arithmetic and communication costs separately. We assume that $n \geq p^{1+\varepsilon}$, where $\varepsilon$ denotes an arbitrary positive constant, which implies a bounded depth of recursion. Under this assumption, the total arithmetic cost of column sort is $\Theta((n / p) \lg n)$, which is optimal for any comparison-based sort. With pipelining, the communication cost of column sort is $\Theta(n / p)$, which is optimal for any sorting algorithm.

To summarize, although we felt that column sort might turn out to be competitive at unusually high loads $\left(n \geq p^{3}\right)$, its mediocre performance at high loads $\left(p^{2} \leq n<p^{3}\right)$, and poor performance at low to moderate loads $\left(p \leq n<p^{2}\right)$ made other alternatives more attractive. Column sort might well be a useful component of a hybrid sorting scheme that automatically selects an appropriate algorithm depending upon the values of $n$ and $p$.

Cubesort. Like column sort, the cubesort algorithm of Cypher and Sanz [9] gives a scheme for sorting $n$ items in a number of "rounds," where in each round the data is partitioned into $n / s$ sets of size $s$ (for some $s, 2 \leq s \leq n$ ), and each set is sorted. (Successive partitions of the data are determined by simple fixed permutations that can be routed just as efficiently as those used by column sort.) The main advantage of cubesort over column sort is that, for a wide range of values of $s$, cubesort requires asymptotically fewer rounds than column sort. In particular, for $2 \leq s \leq n$, column sort (applied recursively) uses $\Theta\left((\lg n / \lg s)^{\beta}\right)$ rounds for $\beta=2 /(\lg 3-1) \approx 3.419$, whereas cubesort uses only $O\left((25)^{\lg ^{*} n-\lg ^{*} s}(\lg n / \lg s)^{2}\right)$ rounds. (The cost of implementing a round is essentially the same in each case.) For $n \geq p^{3}$, cubesort can be implemented without recursion, but requires seven rounds as opposed to four for column sort. For $n<p^{3}$, both cubesort and column sort are applied recursively. For $n$ sufficiently smaller 
than $p^{3}$ (and $p$ sufficiently large), the aforementioned asymptotic bounds imply that cubesort will eventually outperform column sort. However, for practical values of $n$ and $p$, if such a crossover in performance ever occurs, it appears likely to occur at a point where both cubesort and column sort have poor performance relative to other algorithms (e.g., at low to moderate loads).

Nonadaptive Smoothsort. There are several variants of the smoothsort algorithm, all of which are described in [18]. The most practical variant, and the one of interest to use here, is the nonadaptive version of the smoothsort algorithm. The structure of this algorithm, hereinafter referred to simply as "smoothsort," is similar to that of column sort. Both algorithms make progress by ensuring that under a certain partitioning of the data into subcubes, the distribution of ranks of the items within each subcube is similar. The benefit of performing such a "balancing" operation is that after the subcubes have been recursively sorted, all of the items can immediately be routed close to their correct position in the final sorted order (i.e., the subcubes can be approximately merged in an oblivious fashion). The effectiveness of the algorithm is determined by how close (in terms of number of processors) every item is guaranteed to come to its correct sorted position. It turns out that for both column sort as well as smoothsort, the amount of error decreases as $n / p$, the load per processor, is increased.

As noted in the preceding section, for $n \geq p^{3}$, column sort can be applied without recursion. This is due to the fact that after merging the balanced subcubes, every item has either been routed to the correct processor $i$, or it has been routed to one of processors $i-1$ and $i+1$. Thus, the sort can be completed by performing local sorts followed by merge-and-split operations between odd and even pairs of adjacent processors. As a simple optimization, it is more efficient to sort the $i$ th largest set of $n / p$ items to the processor with the $i$ th largest standard Gray code instead of processor $i$. This permits the merge-and-split operations to be performed between adjacent processors.

The main difference between column sort and smoothsort is that the "balancing" operation performed by smoothsort (the cost of which is related to that of column sort by a small constant factor) guarantees an asymptotically smaller degree of error. For this reason, smoothsort can be applied without recursion over a larger range of values of $n$ and $p$, namely, for $n \geq p^{2} \lg p$. Interestingly, the balancing operation of smoothsort is based upon a simple variant of merge-and-split: the "merge-and-unshuffle" operation. Essentially, the best way to guarantee similarity between the distribution of ranks of the items at a given pair $\mathrm{A}$ and $\mathrm{B}$ of adjacent processors is to merge the two sets of items, assign the odd-ranked items in the resulting sorted list to processor A (say), and the even-ranked items to processor B. This effect is precisely that of a merge-and-unshuffle operation. The balancing operation of smoothsort amounts to performing $\lg p$ sets of such merge-and-unshuffle operations, one over each of the hypercube dimensions. As in the case of column sort, there are at least two ways to pipeline the balancing operation in order to take advantage of the CM-2's ability to communicate across all of the hypercube wires at once.

At high loads ( $p^{2} \leq n<p^{3}$ ), we felt that smoothsort might turn out to be competitive with sample sort. Like column sort, however, the performance of smoothsort degrades (relative to that of other algorithms) at low to moderate loads ( $p \leq n<p^{2}$ ), which was the overriding factor in our decision not to implement smoothsort. For un- 
usually high loads $\left(n \geq p^{3}\right.$ ), it is likely that column sort would slightly outperform smoothsort because of a small constant factor advantage in the running time of its balancing operation on the CM-2. It should be mentioned that, for $n \geq p^{1+\varepsilon}$, the asymptotic performance of smoothsort is the same as that of column sort, both in terms of arithmetic as well as communication. Smoothsort outperforms column sort for smaller values of $n / p$, however. For a detailed analysis of the running time of smoothsort, the reader is referred to [18].

Theoretical Results. This subsection summarizes several "theoretical" sorting results-algorithms with optimal or near-optimal asymptotic performance but which remain impractical due to large constant factors and/or nonconstant costs that are not accounted for by the model of computation. In certain instances, a significant additional penalty must be paid in order to "port" the algorithm to the particular architecture provided by the CM-2.

Many algorithms have been developed for sorting on parallel random access machines (PRAMs). The fastest comparison-based sort is Cole's parallel merge sort [6]. This algorithm requires optimal $O(\lg n)$ time to sort $n$ items on an $n$-processor exclusiveread exclusive-write (EREW) PRAM. Another way to sort in $O(\lg n)$ time is to emulate the AKS sorting circuit [1]. In this case, however, the constants hidden by the $O$-notation are large.

If one is interested in emulating a PRAM algorithm on a fixed-connection network such as the hypercube or butterfly, the cost of the emulation must be taken into account. Most emulation schemes are based on routing. Since the cost of sample sort is only about three times the cost of a single routing operation, it seems unlikely that any direct emulation of a PRAM sorting algorithm will lead to a competitive solution.

For the hypercube and related networks such as butterfly, cube-connected cycles, and shuffle-exchange, there have been recent asymptotic improvements in both the deterministic and randomized settings. A deterministic $O\left(\lg n(\lg \lg n)^{2}\right)$-time algorithm for the case $n=p$ is described in [8]. An $O(\lg n)$-time algorithm that admits an efficient bit-serial implementation and also improves upon the asymptotic failure probability of the Reif-Valiant flashsort algorithm is presented in [16]. Unfortunately, both of these algorithms are quite impractical. The reader interested in theoretical bounds should consult the aforementioned papers for further references to previous work.

\section{Appendix B. Probabilistic Analysis of Sample Sort}

This appendix analyzes the sizes of the buckets created by the sample sort algorithm from Section 5. Recall how buckets are created, a method we call Method P. First, each of the $p$ processors partitions its $n / p$ keys into $s$ groups of $n / p s$ and selects one candidate at random from each group. Thus, there are a total of exactly $p s$ candidates. Next, the candidates are sorted, and every sth candidate in the sorted order is chosen to be a splitter. The keys lying between two successive splitters form a bucket. Theorem B.4 shows that it is unlikely that this method assigns many more keys than average to any one bucket.

The proof of Theorem B.4 uses three lemmas, the first two of which are well known in the literature. The first is due to Hoeffding. 
Lemma B.1. Let $X_{i}$ be a random variable that is equal to 1 with probability $q_{i}$ and to 0 with probability $1-q_{i}$, for $i=1,2, \ldots, n$. Let $W=\sum_{i=1}^{n} X_{i}$, which implies that $E[W]=\sum_{i=1}^{n} q_{i}$. Let $q=E[W] / n$, and let $Z$ be the sum of $n$ random variables, each equal to 1 with probability $q$ and to 0 with probability $1-q$. (Note that $E[W]=E[Z]=$ $q n$.) If $k \leq q n-1$ is an integer, then

$$
\operatorname{Pr}[W \leq k] \leq \operatorname{Pr}[Z \leq k]
$$

Our second lemma is a "Chernoff" bound due to Angluin and Valiant [11].

Lemma B.2. Consider a sequence of $r$ Bernoulli trials, where success occurs in each trial with probability $q$. Let $Y$ be the random variable denoting the total number of successes. Then, for $0 \leq \gamma \leq 1$, we have

$$
\operatorname{Pr}[Y \leq \gamma r q] \leq e^{-(1-\gamma)^{2} r q / 2}
$$

Our third lemma shows that Method P can be analyzed in terms of another simpler method which we call Method I. In Method I each key of the $n$ keys independently chooses to be a candidate with probability $p s / n$. For this method, the expected number of candidates is $p s$. The following lemma shows that upper bounds for Method I apply to Method P.

Lemma B.3. Let $S$ be a set of $n$ keys, and let $T$ denote an arbitrary subset of $S$. Let $Y_{P}$ and $Y_{I}$ denote the number of candidates chosen from $T$ by Methods $P$ and $I$, respectively. Then, for any integer $k \leq(|T| p s / n)-1$, we have

$$
\operatorname{Pr}\left[Y_{P} \leq k\right] \leq \operatorname{Pr}\left[Y_{I} \leq k\right]
$$

Proof. Let $\left\{S_{i}\right\}$ be the partition of keys used by Method P, that is, $S=\cup_{i=1}^{p s} S_{i}$ and $\left|S_{i}\right|=n / p s$. Define $T_{i}=S_{i} \cap T$, for $i=1,2, \ldots, p s$. Since $\left|T_{i}\right| \leq\left|S_{i}\right|=n / p s$, under Method $\mathrm{P}$ each set $T_{i}$ contributes one candidate with probability $\left|T_{i}\right| p s / n$, and no candidates otherwise.

Now, define $|T| 0-1$ random variables as follows. For each nonempty $T_{i}$, define $\left|T_{i}\right|$ random variables, where the first random variable is equal to 1 with probability $\left|T_{i}\right| p s / n$ and 0 otherwise, and the remaining $\left|T_{i}\right|-1$ random variables are always 0 .

Call the resulting set of $|T|$ random variables $X_{1}, \ldots, X_{|T|}$ (order is unimportant), and let $Y_{P}$ be the random variable defined by $Y_{P}=\sum_{i=1}^{|T|} X_{i}$. Consequently,

$$
E\left[Y_{P}\right]=\sum_{i=1}^{|T|} E\left[X_{i}\right]=\sum_{i=1}^{p s}\left|T_{i}\right| p s / n=|T| p s / n
$$

and, thus, $Y_{P}$ is the random variable corresponding to the number of candidates chosen from the set $T$ by Method P. 
Define $Y_{I}$ to be the sum of $|T| p s / n$-biased Bernoulli trials. Note that $Y_{I}$ is the random variable corresponding to the number of candidates chosen from the set $T$ by Method I. Hence, by substituting $W=Y_{P}$ and $Z=Y_{I}$ into Lemma B.1, we have

$$
\operatorname{Pr}\left[Y_{P} \leq k\right] \leq \operatorname{Pr}\left[Y_{I} \leq k\right]
$$

for $k \leq E\left[Y_{P}\right]-1=E\left[Y_{I}\right]-1=(|T| p s / n)-1$.

With Lemmas B.2 and B.3 in hand, we are prepared to prove the bound given by (9).

Theorem B.4. Let $n$ be the number of keys in a sample sort algorithm, let $p$ be the number of processors, and let $s$ be the oversampling ratio. Then, for any $\alpha \geq 1+1 / s$, the probability that Method $P$ causes any bucket to contain more than $\alpha n / p$ keys is at most $n e^{-(1-1 / \alpha)^{2} \alpha s / 2}$.

Proof. To prove that no bucket receives more than $\alpha n / p$ keys, it suffices to show that the distance $l$ from any key to the next splitter in the sorted order is at most $\alpha n / p$. We begin by looking at a single key. We have $l>\alpha n / p$ only if fewer than $s$ of the next $\alpha n / p$ keys in the sorted order are candidates. Let $T$ denote this set of $\alpha n / p$ keys. Let $Y_{P}$ denote the number of candidates in $T$, which are chosen according to Method P. Thus, $\operatorname{Pr}[l>\alpha n / p] \leq \operatorname{Pr}\left[Y_{P}<s\right]$.

We can obtain an upper bound on $\operatorname{Pr}\left[Y_{P}<s\right]$ by analyzing Method I instead of Method P, since, by Lemma B.3, any upper bound derived for $\operatorname{Pr}\left[Y_{I} \leq s\right]$ also applies to $\operatorname{Pr}\left[Y_{P}<s\right]$, as long as $s \leq(|T| p s / n)-1$, which holds for $\alpha \geq 1+1 / s$. If the candidates are chosen according to Method I, then the number of candidates in the set $T$ of $\alpha n / p$ keys has a binomial distribution, that is,

$$
\operatorname{Pr}\left[Y_{I}=k\right]=\left(\begin{array}{l}
r \\
k
\end{array}\right) q^{k}(1-q)^{r-k}
$$

where $r=\alpha n / p$ is the number of independent Bernoulli trials, $q=p s / n$ is the probability of success in each trial, and $Y_{I}$ is the number of successes. The probability that fewer successes occur than expected can be bounded using the "Chernoff" bound

$$
\operatorname{Pr}\left[Y_{I} \leq \gamma r q\right] \leq e^{-(1-\gamma)^{2} r q / 2},
$$

which holds for $0 \leq \gamma \leq 1$. Substituting $r=\alpha n / p, q=p s / n$, and $\gamma=1 / \alpha$, we have

$$
\begin{aligned}
\operatorname{Pr}[l>\alpha n / p] & \leq \operatorname{Pr}\left[Y_{P} \leq s\right] \\
& \leq \operatorname{Pr}\left[Y_{I} \leq s\right] \\
& \leq e^{-(1-1 / \alpha)^{2} \alpha s / 2}
\end{aligned}
$$


The probability that the distance from any of the $n$ keys to the next splitter is more than $\alpha n / p$ is at most the sum of the individual probabilities, each of which is bounded by $e^{-(1-1 / \alpha)^{2} \alpha s / 2}$. Since there are $n$ keys, the probability that the distance from any key to the next splitter is greater than $\alpha n / p$ is therefore at most $n e^{-(1-1 / \alpha)^{2} \alpha s / 2}$, which proves the theorem.

\section{References}

[1] M. Ajtai, J. Komlós, and E. Szemerédi. Sorting in $c \log n$ parallel steps. Combinatorica, 3:1-19, 1983.

[2] S. G. Akl. Parallel Sorting Algorithms. Academic Press, Toronto, 1985.

[3] K. Batcher. Sorting networks and their applications. In Proceedings of the AFIPS Spring Joint Computing Conference, volume 32, pages 307-314, 1968.

[4] G. Baudet and D. Stevenson. Optimal sorting algorithms for parallel computers. IEEE Transactions on Computers, 27:84-87, 1978.

[5] G. E. Blelloch. Vector Models for Data-Parallel Computing. MIT Press, Cambridge, MA, 1990.

[6] R. Cole. Parallel merge sort. SIAM Journal on Computing, 770-785, 1988.

[7] T. H. Cormen, C. E. Leiserson, and R. L. Rivest. Introduction to Algorithms. MIT Press, Cambridge, MA, and McGraw-Hill, New York, 1990.

[8] R. E. Cypher and C. G. Plaxton. Deterministic sorting in nearly logarithmic time on the hypercube and related computers. In Proceedings of the 22nd Annual ACM Symposium on Theory of Computing, pages 193-203, May 1990.

[9] R. E. Cypher and J. L. C. Sanz. Cubesort: A parallel algorithm for sorting $N$ data items with $S$-sorters. Journal of Algorithms, 13:211-234, 1992.

[10] W. D. Frazer and A. C. McKellar. Samplesort: A sampling approach to minimal storage tree sorting. Journal of the Association for Computing Machinery, 17(3):496-507,1970.

[11] T. Hagerup and C. Rub. A guided tour of Chernoff bounds. Information Processing Letters, 33(6):305308, 1990.

[12] W. L. Hightower, J. F. Prins, and J. H. Reif. Implementations of randomized sorting on large parallel machines. In Proceedings of the Symposium on Parallel Algorithms and Architectures, June 1992.

[13] J. S. Huang and Y. C. Chow. Parallel sorting and data partitioning by sampling. In Proceedings of the IEEE Computer Society's Seventh International Computer Software and Applications Conference, pages 627-631, November 1983.

[14] L. Johnsson. Combining parallel and sequential sorting on a boolean $n$-cube. In Proceedings of the International Conference on Parallel Processing, pages 444-448, 1984.

[15] F. T. Leighton. Tight bounds on the complexity of parallel sorting. IEEE Transactions in Computers, 34(4):344-354, 1985.

[16] T. Leighton and G. Plaxton. A (fairly) simple circuit that (usually) sorts. In Proceedings of the 31st Annual Symposium on Foundations of Computer Science, pages 264-274, October 1990.

[17] D. Nassimi and S. Sahni. Parallel permutation and sorting algorithms and a new generalized connection network. Journal of the Association for Computing Machinery, 29(3):642-667, 1982.

[18] C. G. Plaxton. Efficient computation on sparse interconnection networks. Technical Report STAN-CS89-1283, Department of Computer Science, Stanford University, September 1989.

[19] J. H. Reif and L. G. Valiant. A logarithmic time sort for linear size networks. Journal of the Association for Computing Machinery, 34(1):60-76, 1987.

[20] S. R. Seidel and W. L. George. Binsorting on hypercubes with $d$-port communication. In Proceedings of the Third Conference on Hypercube Concurrent Computers and Applications, pages 1455-1461. ACM, New York, January 1988.

[21] T. M. Stricker. Supporting the hypercube programming model on mesh architectures (a fast sorter for iWarp tori). In Proceedings of the Symposium on Parallel Algorithms and Architectures, June 1992.

[22] K. Thearling and S. Smith. An improved supercomputer sorting benchmark. In Proceedings Supercomputing '92, pages 14-19, November 1992. 
[23] B. A. Wagar. Hyperquicksort: A fast sorting algorithm for hypercubes. In M. T. Heath, editor, Hypercube Multiprocessors 1987 (Proceedings of the Second Conference on Hypercube Multiprocessors), pages 292-299. SIAM. Philadelphia, PA, 1987.

[24] Y. Won and S. Sahni. A balanced bin sort for hypercube multicomputers. Journal of Supercomputing, 2:435-448, 1988.

[25] M. Zagha and G. E. Blelloch. Radix sort for vector multiprocessors. In Proceedings Supercomputing '91, pages 712-721, November 1991.

Received April 1996, and in final form June 1996. 